\title{
Circuits That Mediate Expression of Signaled Active Avoidance Converge in the Pedunculopontine Tegmentum
}

\author{
Sebastian Hormigo, German Vega-Flores, Victor Rovira, and Manuel A. Castro-Alamancos \\ Department of Neurobiology and Anatomy, Drexel University College of Medicine, Philadelphia, Pennsylvania 19129
}

An innocuous sensory stimulus that reliably signals an upcoming aversive event can be conditioned to elicit locomotion to a safe location before the aversive outcome ensues. The neural circuits that mediate the expression of this signaled locomotor action, known as signaled active avoidance, have not been identified. While exploring sensorimotor midbrain circuits in mice of either sex, we found that excitation of GABAergic cells in the substantia nigra pars reticulata blocks signaled active avoidance by inhibiting cells in the pedunculopontine tegmental nucleus (PPT), not by inhibiting cells in the superior colliculus or thalamus. Direct inhibition of putative-glutamatergic PPT cells, excitation of GABAergic PPT cells, or excitation of GABAergic afferents in PPT, abolish signaled active avoidance. Conversely, excitation of putative-glutamatergic PPT cells, or inhibition of GABAergic PPT cells, can be tuned to drive avoidance responses. The PPT is an essential junction for the expression of signaled active avoidance gated by nigral and other synaptic afferents.

\section{Significance Statement}

When a harmful situation is signaled by a sensory stimulus (e.g., street light), subjects typically learn to respond with active or passive avoidance responses that circumvent the threat. During signaled active avoidance behavior, subjects move away to avoid a threat signaled by a preceding innocuous stimulus. We identified a part of the midbrain essential to process the signal and avoid the threat. Inhibition of neurons in this area eliminates avoidance responses to the signal but preserves escape responses caused by presentation of the threat. The results highlight an essential part of the neural circuits that mediate signaled active avoidance behavior.

\section{Introduction}

To assure survival, animals express evolutionarily conserved defensive behaviors that eliminate harm. Given the opportunity, mice innately flee an environment in response to presentation of a predator, application of a painful unconditioned stimulus (US) (Blanchard et al., 2001; Yilmaz and Meister, 2013; Tovote et al., 2015), or activation of neural circuits that produce aversion, such as in place preference tests (Lammel et al., 2012; Shabel et al., 2012; Tan et al., 2012; Betley et al., 2015; Shang et al., 2015). An escape response is unconditioned rapid locomotion fleeing away from present aversive events or predators toward a safe location.

\footnotetext{
Received Jan. 8, 2019; revised March 16, 2019; accepted March 26, 2019.

Author contributions: M.A.C.-A. designed research; S.H., G.V.-F., V.R., and M.A.C.-A. performed research; S.H., G.V.-F., V.R., and M.A.C.-A. analyzed data; M.A.C.-A. wrote the first draft of the paper; S.H., G.V.-F., V.R., and M.A.C.-A. edited the paper; M.A.C.-A. wrote the paper.

This work was supported by NIH Grants to M.A.C.-A. We thank Ji Zhou and Matthew Andres for technical assistance, and the NIDA drug supply program for providing clozapine- $\mathrm{N}$-oxide. Additional information is available at https://castro-lab.org/.

The authors declare no competing financial interests.

Correspondence should be addressed to Manuel Castro-Alamancos at castrolab@outlook.com.

https://doi.org/10.1523/JNEUROSCI.0049-19.2019

Copyright $\odot 2019$ the authors
}

Through learning processes, a neutral sensory stimulus that reliably signals an upcoming aversive event (capable of evoking an escape response) can be conditioned to elicit locomotion to a safe location before the aversive outcome ensues (avoidance response). In signaled active avoidance conditioning, animals learn to avoid a harmful US by moving between compartments in a cage (shuttling) during an interval signaled by a conditioned stimulus (CS; Mowrer, 1960; Bolles, 1970; Mineka, 1979; LeDoux et al., 2017). Once signaled active avoidance has been acquired, animals typically do not display evident signs of fear during performance, likely because of the predictability and control over the harmful outcome (Kamin et al., 1963; Bolles et al., 1966; Rescorla and Solomon, 1967; Bolles, 1970; Seligman et al., 1973; Mineka and Hendersen, 1985). The active avoidance response is a goaldirected behavior (action) supported by negative reinforcement; the harmful outcome is contingent on the behavior of the animal; if the animal shuttles during the CS presentation, the US does not occur.

Signaled active avoidance provides an opportunity to decipher the neural circuits that mediate the expression of a learned, CS-signaled, locomotor action. Performance of this behavior in well trained animals likely involves the interplay between at least three related neural systems: the structures associated with the 
sensory modality (auditory, somatosensory, or visual) that processes the CS, the structures that drive the conditioned locomotor response, and an intermediate circuit that gates avoidance to the CS. Sensory areas appear to be redundant in CS processing. For instance, the sensory thalamus only critically mediates signaled active avoidance when the superior colliculus is not present and vice versa (Cohen and Castro-Alamancos, 2007, 2010a). The superior colliculus responds to the CS, ramps up its firing during signaled avoidance responses (Cohen and Castro-Alamancos, 2010b), and drives escape responses when optogenetically stimulated (Shang et al., 2015; Wei et al., 2015). Furthermore, the substantia nigra pars reticulata ( $\mathrm{SNr}$ ), a main GABAergic output nucleus of the basal ganglia that projects to the superior colliculus, controls the expression of signaled active avoidance responses (Hormigo et al., 2016). In particular, increasing $\mathrm{SNr}$ cell firing, to levels that do not interfere with escape responses driven by the US, abolishes active avoidance responses driven by the CS. However, the nucleus contacted by SNr cells that is responsible for controlling signaled active avoidance is unknown.

The SNr projects to the superior colliculus (Bentivoglio et al., 1979; Di Chiara et al., 1979; Chevalier and Deniau, 1990), pedunculopontine tegmental nucleus (PPT; Mena-Segovia et al., 2004; Mena-Segovia and Bolam, 2017), certain thalamic nuclei (Di Chiara et al., 1979; Gulcebi et al., 2012), and leave collaterals within substantia nigra pars compacta (Tepper et al., 1995; Richards et al., 1997). Using cell-type-specific optogenetics, we identified the PPT as a critical junction for the expression of this learned, CS-signaled, locomotor action. The results provide a framework for how signaled active avoidance responses can be regulated by diverse brain regions.

\section{Materials and Methods}

Experimental design and statistical analysis. All procedures were reviewed and approved by the Animal Care Committee of Drexel University and conducted in adult ( $>8$ weeks) male and female mice. The results from both sexes were combined because there is no sex difference in the behavior measured for the strains used. Thus, comparison of the initial performance sessions (Sessions $3-6)$ between males $(n=42)$ and females $(n=37)$, including all the mice in this study, revealed no significant differences in avoidance rate (ANOVA, $F_{(1,268)}=0.23 ; p=0.63$ ), latency $\left(F_{(1,268)}=0.02 ; p=0.89\right)$ or intertrial crossings $\left(F_{(1,268)}=0.0004 ; p=\right.$ $0.98)$. There were also no significant differences between both sexes when the initial three learning sessions (Sessions 1-3) were compared.

All experiments involved a repeated-measures design in which the mice or cells serve as their own controls. All conclusions are based on within-subjects comparisons; independent comparisons between different groups of mice were not performed (other than the sex comparison shown above). We tested for a main effect [light or clozapine- $N$-oxide $(\mathrm{CNO})]$ using a two-way mixed-design ANOVA followed by comparisons with Tukey's test. In the mixed-design ANOVA, the repeatedmeasures factor was the light (with as many levels as conditions tested) or the CNO (2 levels) effect and the other factor was the animals' sessions. Tukey tests were conducted for the repeated-measures factor when the within-subjects effect ( $F$ value) was statistically significant at a level of $p<0.01$. Optogenetic experiments consist of a balanced design wherein the tested light and control conditions are randomly distributed within the same session, which is repeated on multiple days. Thus, all comparisons are between conditions presented within the same session. We highlight results with significance levels of at least 0.01 . To test for equivalency between auditory CS (ACS) and LCS alone trials we first used the mixed ANOVA Tukey's to establish that the trials were not significantly different $(p>0.01)$. This was followed by an equivalence paired test in which the upper boundary for equivalence was set at $20 \%$.

Power analysis was conducted with both OriginLab Pro and GLIMMPSE using the measured means difference variability. It revealed that three animals in which we conducted five identical daily sessions per animal ( 15 sessions) was sufficient to detect a $\sim 20 \%$ change in avoidance rate with a power of $0.99(p<0.05)$. This was used as the bare minimum number of animals and sessions per group.

The experiment timeline included the following sequence of phases: AAV injections (unless mice natively expressed opsins), active avoidance learning phase, optical fiber implantation (except for DREADDs), active avoidance testing phase, and in vivo (anesthetized) or slice (in vitro) recordings followed by histology. In mice subjected to AAV injections (optogenetics and DREADDs), the active avoidance testing phase commenced 3 weeks after AAV injections. In mice subjected to optogenetics, optical fiber implantation occurred 5-6 d before commencing the active avoidance testing phase, which lasted 3-5 weeks with daily (weekday) sessions. In mice subjected to DREADDs, the active avoidance testing phase lasted 6 weeks and involved 1 week ( $4-5$ daily sessions) of saline (control) sessions followed by 1 week of $\mathrm{CNO}$ sessions, which alternated three times.

To enable rigorous approaches, we maintain a local server with a central database accessed through a wiki that logs all details and metadata related to the experiments, including all information about animals and details about surgical procedures, behavioral sessions, electrophysiological recordings, histology, and scripts used for analyses. Moreover, during daily behavioral sessions or electrophysiological recordings, experiments are run automatically by computers using preset parameters that are automatically logged for reference during analysis. Analyses are performed using scripts that automate all aspects of data analysis from access to metadata and data files to population statistics and graph generation (scripts and metadata will be made accessible through our website or by request).

Surgeries. Optogenetics experiments and DREADDs experiments involved bilaterally injecting a volume of $0.3-0.4 \mu \mathrm{l}$ of AAVs per site during isoflurane anesthesia $(\sim 1 \%)$, except for animals that innately express opsins. Animals received carprofen after surgery. The stereotaxic coordinates for injection sites (in $\mathrm{mm}$ from bregma, lateral from the midline, ventral from the bregma-lambda plane) are as follows: $\mathrm{SNr}$ (3.3 posterior; $1.4-1.5 ; 4-4.1$ ), superior colliculus (4 posterior; $1 ; 1.75$ ), and PPT ( 4.7 posterior; $1.25 ; 3.1$ entering in the posterior direction at a $20^{\circ}$ angle).

In optogenetics experiments, a dual optical fiber ( $200 \mu \mathrm{m}$ in diameter $)$ was implanted bilaterally during isoflurane anesthesia at the previously mentioned coordinates and held in place with a combination of screws, cyanoacrylate, and dental cement. Bilateral optical fibers were implanted in the injection site or in the projection site of the AAV injection site. The stereotaxic coordinates for the implanted optical fibers are as follows (in $\mathrm{mm}$ ): VL/VM thalamus ( 1.2 posterior; $1-1.25 ; 3.2)$, superior colliculus (4 posterior; $1-1.5 ; 1.5-1.8$ ), and PPT (4.7 posterior; 1.25 ; 3.1 entering in the posterior direction at a $20^{\circ}$ angle). The coordinate ranges reflect different animals that were combined together because the slight coordinate differences produced similar effects.

Active avoidance. Mice were trained in the active avoidance task using procedures similar to those described previously for rats and mice (Cohen and Castro-Alamancos, 2007, 2010b; Hormigo et al., 2016). Mice were trained before reaching the testing phase; learning occurred during 4-6 daily sessions (50 trials per session) after AAV injections and before optical fiber implantation (see timeline in the previous section). All reported active avoidance data were gathered during the testing phase.

During an active avoidance session, mice are placed in a standard shuttle box $(16.1 \times 6.5$ inches $)$ that has two compartments separated by a partition with side walls forming a doorway that the animal has to traverse to shuttle between compartments. For active avoidance training during the learning phase, a trial consists of a $7 \mathrm{~s}$ avoidance interval followed by a $10 \mathrm{~s}$ escape interval. During the avoidance interval, an ACS ( $8 \mathrm{kHz}, \sim 85 \mathrm{~dB}$ tone) is presented for the duration of the interval or until the animal produces a conditioned response (avoidance response) by moving to the adjacent compartment, whichever occurs first. If the animal avoids, the CS ends, the escape interval is not presented and the trial terminates. However, if the animal does not avoid, the escape interval ensues consisting of white noise plus a mild scrambled electric footshock $(0.3 \mathrm{~mA})$ delivered through the grid floor of the occupied half of the shuttle box. This US readily drives the animal to move to the adjacent compartment (escape response), at which point the US terminates, end- 
ing the escape interval and the trial. Each trial is followed by an intertrial interval (duration is randomly distributed; $25-45 \mathrm{~s}$ range) during which the animal awaits the next trial and is free to cross between compartments (intertrial crossings). The main variables representing task performance are the percentage of avoidances (avoids) and the response latency from CS onset (time at which the animal enters the safe compartment). The percentage of escape responses (escapes) is equal to 100 minus the percentage of avoidances, because all animals in this study escaped every trial. The response latency reflects the overall latency (avoidance and escape latencies); response latencies $>7 \mathrm{~s}$ are escape latencies from trial onset.

Optogenetics during active avoidance. The implanted dual optical fibers were connected to patch cables using sleeves. A black aluminum cap covered the head implant and completely blocked any light exiting at the ferrules junction. Furthermore, the experiments are conducted in a brightly lit cage that makes it difficult to detect any light. The other end of the patch cables was connected to a dual light swivel (Doric Lenses) that was coupled to a blue laser $(450 \mathrm{~nm}, 80 \mathrm{~mW})$ or a green laser $(520 \mathrm{~nm}$, $100 \mathrm{~mW}$ ). All optogenetics experiments involved a repeated-measures design in which different trials are compared within the same session (50-150 trials per session). Half of the trials in a session are control trials (e.g., ACS trials) and the other half are optogenetics trials (ACS+LCS or LCS alone trials) that used different light stimuli. The order of the trials is randomly distributed within a session but each block of 10 trials includes 5 control trials. The blue light stimuli used during optogenetics trials included continuous pulses and trains of 1 ms pulses at 2, 5, 10, 20, 40, 66, and $100 \mathrm{~Hz}$. Green light was always continuous.

On the first optogenetics ChR2 session, we tested different blue light powers up to a maximum of $\sim 2 \mathrm{~mW}$ (in several experiments this maximum was $3.4 \mathrm{~mW}$ ) and selected the maximum power that did not interfere with the ability of the animal to escape the US during any of the light patterns used. In most cases, when the regular maximum blue light power did not interfere with the ability of the animal to avoid or escape the US, we conducted additional sessions using a higher blue light power of $\sim 6 \mathrm{~mW}$; noted as high-power. Continuous green light was applied at a power of $\sim 25 \mathrm{~mW}$. In some cases, different green light powers $(4,16$, 35, and $45 \mathrm{~mW}$; see Fig. 11) were compared within the same sessions. Power is regularly measured by flashing the connecting patch cords onto a light sensor, with the sleeve on the ferrule. Different light powers and patterns (continuous and train frequencies) were tested because we expected the optogenetic effects to be highly dependent on these variables.

Video tracking. Animals were video tracked (30 FPS) during most active avoidance sessions that used optogenetics. The tracking followed color markers located on the head connector above the nose and between the ears. Several movement (tracking) measures were derived during active avoidance. Distance was the number of pixels crossed by the animal in its trajectory during the avoidance and escape intervals of a trial (trial distance) or during the intertrial interval (intertrial distance). Displacement was the number of pixels in a straight line between the position of the animal at trial start and the position of the animal at trial end (when the animal avoided or escaped). Pixel measures were converted to $\mathrm{cm}$ using calibrations. Trial speed was the trial distance divided by the response latency. Intertrial speed was the intertrial distance divided by the intertrial interval duration. Trial velocity was the displacement divided by the response latency.

Water licking task. After active avoidance sessions, a group of Vgat$\mathrm{SNr}$-ChR2 mice with optical fibers in PPT were water-restricted and subjected to daily sessions in which the animals licked on a spout (computer-monitored lickometer) to obtain water. During the licking sessions, blue light was delivered ( $3 \mathrm{~s} \mathrm{OFF} / 3 \mathrm{~s} \mathrm{ON}$ ) in the PPT using continuous pulses or trains of $1 \mathrm{~ms}$ pulses $(40-66 \mathrm{~Hz})$ at the same blue light power that blocked avoidance responses in each animal.

$D R E A D D s$. In contrast to optogenetics, DREADDs modulate neural activity in a time unselective manner. We used DREADDs as a supplement to optogenetics experiments in superior colliculus and PPT. To excite GABAergic cells, a Cre-inducible AAV [AAV5-hSyn-DIO$\mathrm{hM} 3 \mathrm{D}(\mathrm{Gq})$-mCherry; UNC Vector Core] was bilaterally injected in the superior colliculus or PPT of Vgat-cre mice (Vgat-SC-hM3Dq or VgatPPT-hM3Dq). To inhibit GABAergic cells, a Cre-inducible AAV [AAV5-
hSyn-DIO-hM4D(Gi)-mCherry; UNC Vector Core] was bilaterally injected in the superior colliculus or PPT of Vgat-Cre mice (Vgat-SChM4Di or Vgat-PPT-hM4Di). To nonspecifically inhibit neurons, an AAV with an hSyn promoter [AAV8-hSyn-hM4D(Gi)-mCherry; UNC Vector Core] was bilaterally injected in the superior colliculus or PPT of Vgat-Cre or C57BL/6J mice (hSyn-SC-hM4Di or hSyn-PPT-hM4Di). Mice were initially trained in five sessions (50 trials per daily session). Afterward, in successive daily sessions, animals were subjected to either saline or $\mathrm{CNO}(20 \mathrm{mg} / \mathrm{kg}$, i.p.) injections $45 \mathrm{~min}$ before active avoidance performance (ACS trials). As in our previous study in $\mathrm{SNr}$ (Hormigo et al., 2016), a series of four consecutive saline sessions were followed by a series of four consecutive $\mathrm{CNO}$ sessions and this sequence was repeated three times.

In vivo recordings to test optogenetics. In animals that expressed ChR2 in $\mathrm{SNr}$ cells because of injection of an AAV in the $\mathrm{SNr}$, we tested the effect of blue light applied in the superior colliculus on neural activity recorded in the superior colliculus (and also in PPT in a few cases). After induction of anesthesia with urethane $(1.5 \mathrm{~g} / \mathrm{kg}$, i.p), animals were placed in a stereotaxic frame. Skin incisions and frame contacts with the skin were injected with lidocaine (2\%). Craniotomies and incisions of the dura were made over the target structures (superior colliculus and in some cases PPT) as necessary. Body temperature was automatically maintained constant with a heating pad at $37^{\circ} \mathrm{C}$. The level of anesthesia was monitored with limb withdrawal reflexes. It was kept constant at about stage III/3 (i.e., absence of pinch withdrawal reflex, absence of whisker movements) using supplemental doses. Two tungsten electrodes (1-5 M $\Omega$ ) were moved independently into the intermediate layers of the superior colliculus (and in some cases PPT) on the same side of the brain to record single units or multiunits. An optical fiber was lowered into the superior colliculus on the same side of the brain at the above mentioned coordinates for optical fibers implanted in superior colliculus. Trains of $1 \mathrm{~ms}$ pulses $(2-100 \mathrm{~Hz})$ and continuous pulses of blue light were delivered though an optical fiber $(200 \mu \mathrm{m})$ coupled to an LED with a patch cable ( $1.5 \mathrm{~mW}$; trains and continuous pulses lasted $1 \mathrm{~s}$ ). The light was applied in the superior colliculus while recording unit activity in the superior colliculus and PPT. Each trial was repeated at least 30 times and included different optogenetics stimuli presented in a sequence within the same trial (each $1 \mathrm{~s}$ stimulus separated by $5 \mathrm{~s}$ ) so that a comparison between different light stimuli is done under equal conditions of the recorded cell.

In vitro (slice) recordings to test optogenetics. For slice preparation, adult mice were deeply anesthetized with an overdose of ketamine. Upon losing all responsiveness to a strong tail pinch, the animal was decapitated and the brain was rapidly extracted. Slices ( $400 \mu \mathrm{m}$ thick) were cut in the coronal or sagittal plane using a vibratome at the level of $\mathrm{SNr}$ and PPT. Slices were transferred to an interface chamber where they were bathed constantly $(1-1.5 \mathrm{ml} / \mathrm{min})$ with artificial CSF (ACSF) at $32.5^{\circ} \mathrm{C}$. The ACSF contained the following (in $\mathrm{mm}$ ): $1260 \mathrm{NaCl}, 3 \mathrm{KCl}, 1.25$ $\mathrm{NaH}_{2} \mathrm{Po}_{4}, 26 \mathrm{NaHCO}_{3}, 10$ dextrose, $2 \mathrm{MgSO}_{4} 7 \mathrm{H}_{2} \mathrm{O}, 2 \mathrm{CaCl}_{2}, 2 \mathrm{H}_{2} \mathrm{O}$. Blind whole-cell recordings were obtained from the superior colliculus and PPT using patch electrodes of 4-10 $\mathrm{M} \Omega$ impedance. The electrodes were filled with internal solution containing the following (in mM): 135 K-gluconate, $4 \mathrm{KCl}, 2 \mathrm{NaCl}, 0.2 \mathrm{EGTA}, 10$ Tris-phosphocreatine, 0.3 trisGTP, 10 HEPES, 4 MgATP (290 mOsm). Each slice was imaged using a compound fluorescent microscope to reveal the parts of the brain that contained labeled cells/fibers. This also allowed to verify the correct placement of AAV injections. At the end of each recording, the slice was imaged again to record the location of the whole-cell recording electrodes. The internal solution contained neurobiotin $(0.2 \%)$ to label the recorded cells, which were reconstructed if successfully labeled.

In slice experiments, an optical fiber $(200 \mu \mathrm{m})$ coupled to an LED with a patch cable was used to apply pulses of blue $(470 \mathrm{~nm}, 1.7 \mathrm{~mW})$ or green $(530 \mathrm{~nm}, 1.2 \mathrm{~mW})$ light at the whole-cell recording site. Per trial, trains of $1 \mathrm{~ms}$ pulses $(5-100 \mathrm{~Hz})$ and continuous pulses of light were delivered (500 ms duration at least $4 \mathrm{~s}$ apart) in a sequence so that a comparison between different light stimuli is done under equal conditions of the recorded cell. The effect of light on cell firing was tested both on spontaneous firing (if present) at resting $V_{\mathrm{m}}$ and on firing induced by an $800 \mathrm{~ms}$ positive current pulse triggered $200 \mathrm{~ms}$ before light onset. To confirm the 
GABAergic nature of evoked IPSPs, a $\mathrm{GABA}_{\mathrm{A}}$ receptor antagonist was added to the ACSF (bicuculline methiodide, $10 \mu \mathrm{M}$ ).

Histology. After each slice experiment, the slices were placed in fixative (formalde-fresh solution buffered, Fisher Scientific), later cryoprotected with sucrose $(30 \%)$ and resectioned on a cryostat $(80 \mu \mathrm{m})$. Sections that contain neurobiotin-filled cells are incubated in $0.2 \%$ Triton X-100 and $2 \%$ goat serum followed by Dylight594-streptavidin. Sections are then mounted, coverslipped with DAPI mounting media, and photographed using a fluorescent microscope. Cells were reconstructed using Neurolucida (Microbrightfield).

If animals did not undergo slice procedures, mice were deeply anesthetized with an overdose of ketamine and upon losing all responsiveness to a strong tail pinch, the animal was decapitated and the brain was rapidly extracted and placed in fixative. The brain was sectioned $(100 \mu \mathrm{m}$ sections) in the coronal or sagittal planes. Sections were mounted on slides, coverslipped with DAPI mounting media, and photographed using a fluorescent microscope. The location of the tips of the implanted optical fibers was marked on 3D (coronal or sagittal) atlases generated in our laboratory. Thus, the sections containing the visible tracks of the optical fiber were matched with the 3D atlas sections and then the location of the track ending was marked (see Fig. 2).

\section{Results}

Active avoidance and escape responses

Mice are tested in a standard two-way active avoidance procedure in a shuttle-box ( 2 compartment cage that the animal can shuttle between). A trial consists of consecutive avoidance (7 s) and escape (10 s) intervals followed by a random intertrial interval (25-45 s). The avoidance interval is signaled by a CS; if the animal shuttles during the avoidance interval (avoidance response), the escape interval is omitted and the intertrial interval begins. During the escape interval, a mild foot-shock and white noise are presented; if the animal shuttles during the escape interval (escape response), the intertrial interval begins. During the intertrial interval the animal is free to shuttle between compartments (intertrial crossings).

Mice were tested with different types of trials presented randomly within the same daily session. In ACS trials, the avoidance interval is signaled by an auditory CS (ACS, $8 \mathrm{kHz}$ tone at $\sim 85$ $\mathrm{dB})$. In ACS + LCS trials, the avoidance interval is signaled by the same ACS as in ACS trials and optogenetic light is simultaneously delivered into the brain bilaterally. The light is either a continuous pulse (cont) or a train of $1 \mathrm{~ms}$ pulses at specific frequencies. In LCS alone trials, the avoidance interval is signaled only by light delivered into the brain. In ACS + LCS or LCS alone trials, the light persists during the escape interval. In No CS trials, the avoidance interval is not signaled by any stimulus. Statistical comparisons are repeated measures between the trials tested within a session. Significant statistical differences versus ACS trials $(p<>0.01)$ are indicated in each figure. The purpose of ACS + LCS sessions is to compare ACS + LCS trials versus ACS trials to determine whether the optogenetic manipulation has any effect on avoidance responses driven by the ACS. The purpose of LCS alone sessions is to compare LCS alone trials versus ACS trials to determine whether the optogenetic manipulation is capable of driving avoidance responses as effectively as the ACS. Thus, in ACS alone sessions, we indicate nonsignificant differences $(p>0.01)$ between LCS alone trials and ACS trials to highlight optogenetics stimuli that were equivalent to the ACS in driving avoidance responses.

During task performance, the position of the animal is video tracked (Fig. 1A) to derive movement measures. Figure 1, $B$ and $C$, shows the instantaneous speed of a group of mice $(n=36)$ during ACS trials $(n=11,071)$ in which the animals either avoided ( $84.9 \%$ avoids; $n=9397)$ or escaped ( $15.1 \%$ escapes; $n=$
1674) the US. The speed is either aligned to the onset of the trial (Fig. $1 B$ ) or the occurrence of the response (Fig. 1C). The slower speed from trial onset during avoids compared with escapes (Fig. $1 B$ ) reflects the fact that animals avoid at different times during the avoidance interval, but they escape the US rapidly after it is presented. When the speeds are aligned by the response time (when the animal crosses into the safe compartment; Fig. 1C), it is apparent that animals escape with a peak instantaneous speed that is about twice as fast compared with when they avoid. Otherwise, avoids and escapes are similar locomotion responses toward the safe compartment.

\section{Excitation of SNr fibers in PPT blocks avoidance responses}

Excitation of GABAergic cells within SNr blocks active avoidance (Hormigo et al., 2016), but the target(s) where SNr cells produce this effect is unknown. Because SNr cells project mainly to the thalamus, superior colliculus and PPT, we stimulated SNr fibers in these locations to determine which $\mathrm{SNr}$ targets block avoidance responses. Figure $1 D$ shows a schematic of the pathways manipulated in these experiments. Vgat-Cre mice were injected with a Cre-inducible AAV [AAV5-EF1a-DIO-hChR2(H134R)eYFP; UPenn Vector Core] in the SNr to express ChR2 in SNr GABAergic cells (Vgat-SNr-ChR2), and implanted with bilateral optical fibers in the VL/VM thalamus, superior colliculus or PPT. Superior colliculus and thalamus optical fibers were inserted perpendicular to the bregma-lambda plane. PPT optical fibers entered at a $20^{\circ}$ angle in the posterior direction and targeted the dorsal and posterior portions of PPT to be well separated from $\mathrm{SNr}$ (Fig. 2A). Figure 2, $B$ and $C$, show the location of implanted optical fiber endings in the superior colliculus and PPT (Movies $1,2)$.

Excitation of SNr fibers in the VL/VM thalamus (nigrothalamic fibers) with blue light (3.4 $\mathrm{mW}$, range: 3.4) in ACS+LCS trials had no effect on avoidance responses compared with ACS trials (Fig. 3A, black open squares; 24 sessions in 3 mice) and was ineffective at driving avoidance responses in LCS alone trials (Fig. $3 C$, black open squares; 39 sessions in 3 mice). The percentage of avoidance responses in LCS alone trials was similar to No CS trials. Thus, the projections from SNr to thalamus do not affect active avoidance; they may mediate other roles of $\mathrm{SNr}$ during behavior (Lalive et al., 2018).

Excitation of SNr fibers in the superior colliculus (nigrotectal fibers) with blue light (3.4 mW, range: 3.4 ) produced a significant suppression of avoidance responses in ACS + LCS trials that used trains at $>10 \mathrm{~Hz}$ (Tukey, $p=0.001, p<0.00001, p<0.00001$, and $p<0.00001 ; 20,40,66$, and $100 \mathrm{~Hz}$ vs ACS), but not in trials that used continuous light (Fig. $3 A$, green open circles; 31 sessions in 4 mice). Video tracking (Fig. $3 B$ ) revealed that trial speed and trial velocity were suppressed in ACS + LCS trials that used blue light trains at 66 and $100 \mathrm{~Hz}$ (trial speed: $p=0.003$ and $p=$ 0.01 ; trial velocity: $p=0.0007$ and $p=0.01 ; 66$ and $100 \mathrm{~Hz}$ vs ACS), but trial speed actually increased slightly with continuous blue light $(p=0.01)$. When avoidance responses were suppressed by blue light trains, trial velocity (movement toward the safe compartment) was more strongly suppressed than trial speed (overall movement). Excitation of SNr fibers during LCS alone trials in superior colliculus was highly effective at driving avoidance responses but only for trials that used continuous blue light $(p=0.4)$ or trains at $100 \mathrm{~Hz}(p=0.8$; Fig. $3 C$, green open circles; 33 sessions in 4 mice). Note that in LCS alone trials we highlight nonsignificant differences compared with ACS to indicate that the optogenetic LCS stimulus was not significantly different compared with the ACS. Video tracking (Fig. 3D) revealed that trial 


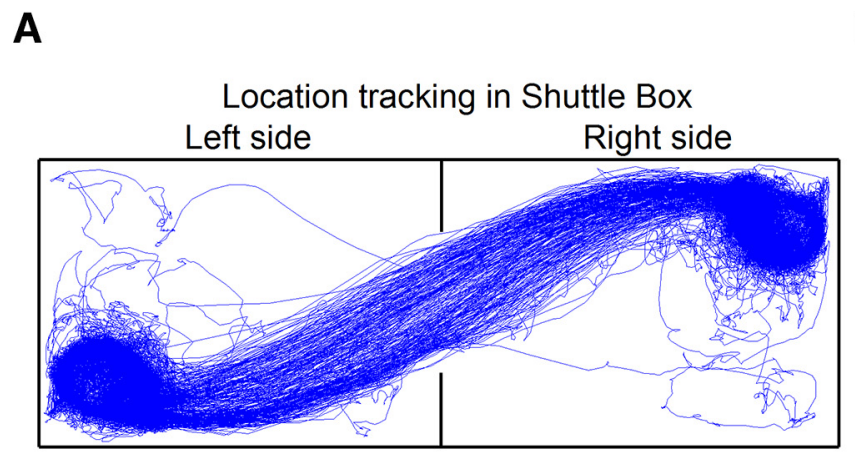

D

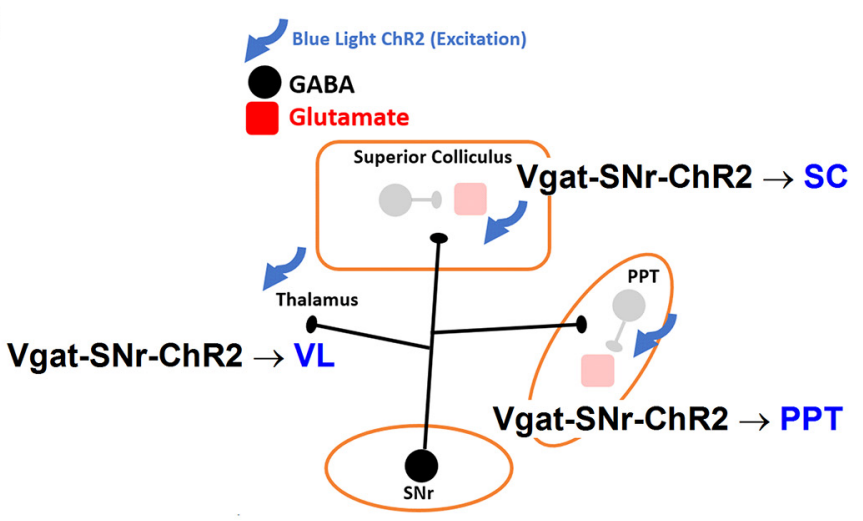

B

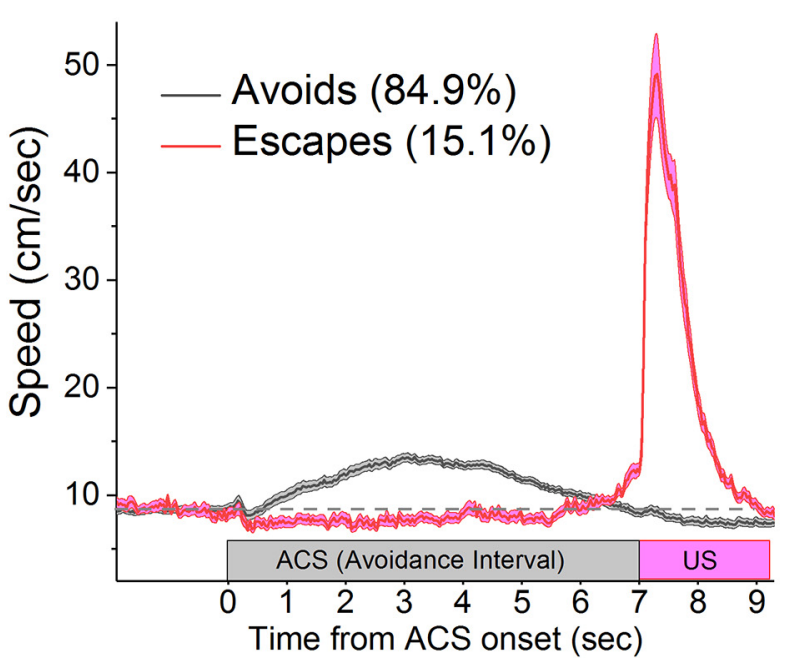

C

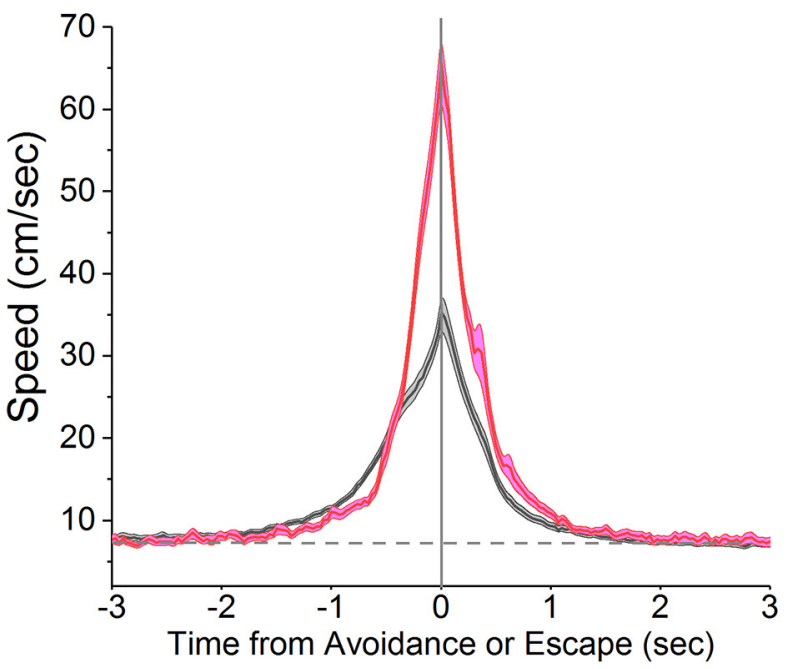

Figure 1. Avoidance and escape responses during active avoidance. $\boldsymbol{A}$, Video tracking showing the position of an animal performing active avoidance in a shuttle box. $\boldsymbol{B}$, Speed during active avoidance performance obtained from a group of mice $(n=36)$ performing ACS trials. The instantaneous speed ( \pm SEM) is plotted versus the onset of ACS trials for avoidance responses (avoids; black) and escape responses (escapes; red). $\boldsymbol{C}$, The instantaneous speed ( \pm SEM) is plotted versus the occurrence of avoidance or escape responses for the data in $\boldsymbol{B}$. $\boldsymbol{D}$, Schematic of the circuits manipulated in this study highlighting the GABAergic pathways activated in the experiments shown in Figure 3. SC, Superior colliculus; VL, ventrolateral/ventromedial thalamus.

velocity during continuous blue light was equivalent $(p=0.24)$ to ACS trials. Thus, excitation of nigrotectal fibers in superior colliculus with continuous blue light is a highly effective CS to drive avoidance responses, but the same stimulation delivered as trains at $\sim 66 \mathrm{~Hz}$ robustly blocks avoidance responses.

Excitation of SNr fibers in PPT (nigrotegmental fibers) with blue light $(0.8 \mathrm{~mW}$, range: $0.4-1.5)$ produced a strong suppression of avoidance responses in ACS +LCS trials for all trains $>5$ $\mathrm{Hz}$ (Tukey, $p<0.00001$ ), including continuous light (Fig. 3A, blue closed circles; 69 sessions in 5 mice). Video tracking (Fig. $3 B$ ) revealed that both trial speed and trial velocity were suppressed in ACS + LCS trials that used blue light trains $>20 \mathrm{~Hz}$ (trial speed: $p=0.0003, p=0.0001$, and $p=0.009$; trial velocity: $p<0.00001$; 60,66 , and $100 \mathrm{~Hz}$ vs ACS), whereas intertrial speed was unaffected. Trial velocity was more strongly suppressed than trial speed for all blue light stimuli $>5 \mathrm{~Hz}$, including continuous light $(p<0.00002)$; trial speed was not suppressed by $10 \mathrm{~Hz}$ or continuous blue light, indicating that the suppression by these optogenetic stimuli was specifically of movement toward the safe compartment. Because stimulation of nigrotegmental fibers in PPT produced a very strong and broad suppression of avoidance responses driven by the ACS, LCS alone trials were not tested in these animals.

To determine whether the level of excitation of nigrotegmental fibers that blocks active avoidance responses impairs other basic behaviors, after the end of active avoidance sessions, a group of Vgat-SNr-ChR2 animals with optical fibers in PPT were water-restricted and tested on water licking from a spout (lickometer). During the licking sessions (26 sessions in 3 mice), blue light was delivered $(3 \mathrm{~s} \mathrm{OFF} / 3 \mathrm{~s} \mathrm{ON})$ in the PPT using trains of 1 ms pulses $(40-66 \mathrm{~Hz})$ at the same blue light power that blocked avoidance responses in each animal. Figure 4 shows that during the blue light trains, which are the most effective optogenetic stimuli to block active avoidance in this group, there were only minor effects on licking. Typically, in some of the earlier trials within each session, the animals stopped licking transiently during the onset of the light (i.e., during the first second of the $3 \mathrm{~s}$ light $\mathrm{ON}$ period) so that the overall reduction in licking was significant but only during the initial $1500 \mathrm{~ms}$ of the light ON period (Tukey, $p<0.0001$ ) and this reduction was modest $(\sim 20 \%)$. After this transient effect, the animals continued licking during the same trial despite the presence of blue light in PPT. The effect appeared to be an orienting response by which the animal moved the head out of the lick port and then moved it back in and continued licking. Thus, levels of nigrotegmental fiber activation that completely block signaled avoidance responses have only relatively minor effects on licking behavior in the same animals.

To test for nonspecific effects of the light, a group of No Opsin controls were implanted with bilateral optical fibers in PPT, superior colliculus, or $\mathrm{SNr}$ and subjected to ACS+LCS or LCS alone sessions. The blue and green light stimulation patterns and 

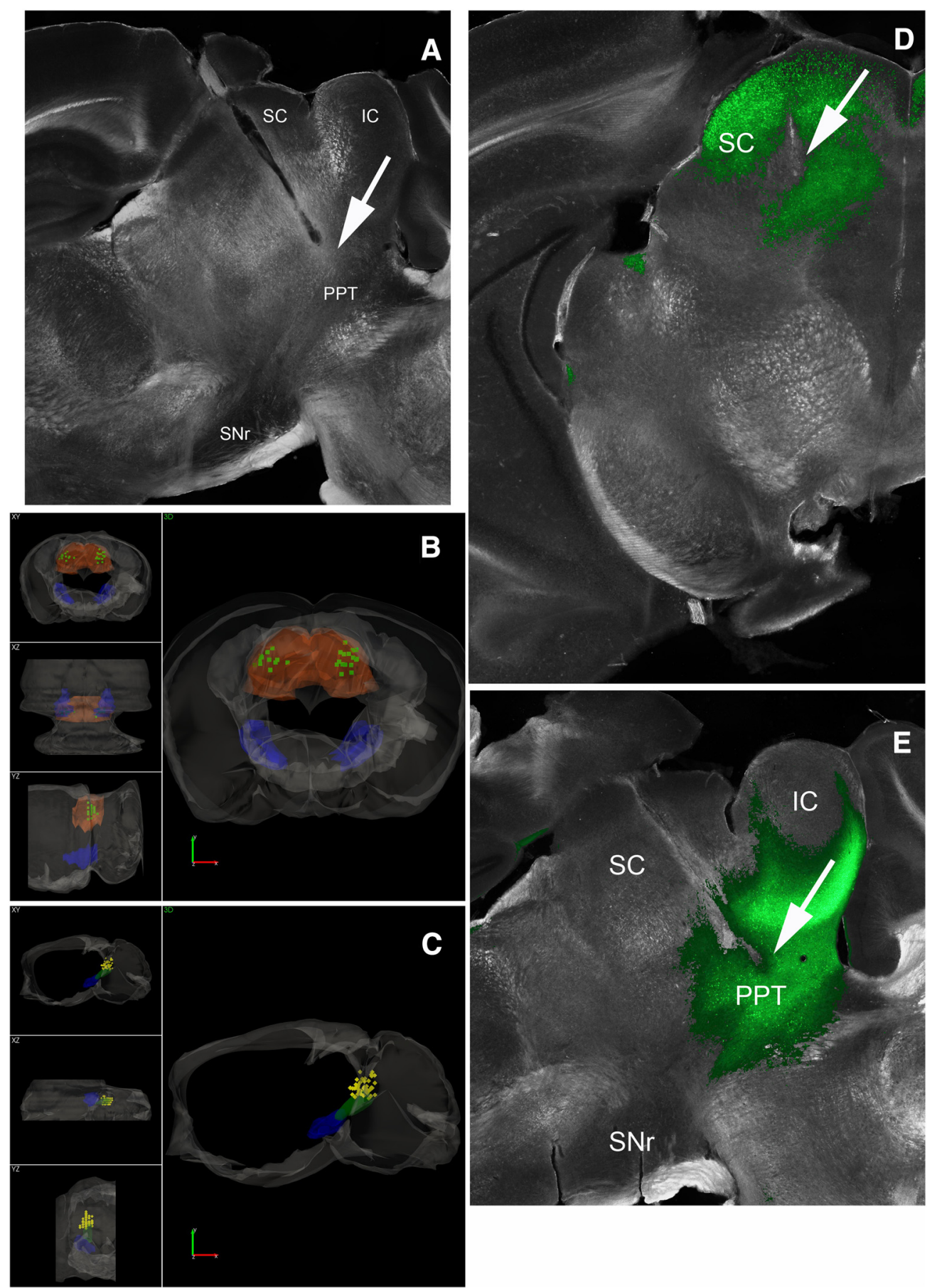

Figure 2. Locations of implanted optical fibers in PPT and superior colliculus. $A$, Dark-field image of a parasagittal section ( $\sim 1 \mathrm{~mm}$ lateral from the midline) showing the trajectory of an optical fiber track coursing toward PPT. The PPT optical fibers are inserted at a $20^{\circ}$ angle in the posterior direction. Stimulation of GABAergic nigrotegmental fibers with this optical fiber using blue light trains $(66 \mathrm{~Hz}$ ) at a power $<1 \mathrm{~mW}$ completely abolished signaled active avoidance without affecting escape responses to the US (Fig. 3). $\boldsymbol{B}, 3 \mathrm{D}$ reconstruction of optical fiber track endings in the superior colliculus (SC; green squares) for brains cut in the coronal plane. SNr and SC are filled in semitransparent blue and orange, respectively. Scale bars, $1.0 \mathrm{~mm}$. A video of the $3 \mathrm{D}$ reconstruction is provided (Movie 1). C, 3D reconstruction of optical fiber track endings in the PPT (yellow diamonds) for brains cut in the sagittal plane. SNr and PPT are filled in semitransparent blue and green, respectively. The bilateral optical fibers from both hemispheres are shown in the same hemisphere. Scale bars, $1.2 \mathrm{~mm}$. A video of the 3D reconstruction is provided (Movie 2). D, Photomicrograph showing eYFP fluorescence in the superior colliculus of a CaMKII-SC-ChR2 mouse (coronal section). Image blends a dark-field image of the section with the green channel of the fluorescent image. The arrow points to the optical fiber tract implanted in superior colliculus. E, Photomicrograph showing eYFP fluorescence in the PPT of a CaMKII-PPT-ChR2 mouse (parasagittal section). Image blends a dark-field image of the section with the green channel of the fluorescent image. The arrow points to the optical fiber tract implanted in PPT. IC, Inferior Colliculus.

highest powers used in the study were combined into one group (Fig. $3 A, C$, open triangles). We found that there was no significant difference in any of the measured variables between ACS + LCS trials and ACS trials in this group of mice (avoidance rates were $92.4 \pm 1.2 \%$ for ACS trials vs $92.12 \pm 1.7 \%$ for
ACS + LCS trials; Tukey, $p=0.9 ; 27$ sessions from 5 mice). The same comparison was conducted for LCS alone trials. No Opsin animals receiving continuous green or blue light avoided the US at much higher rates during ACS trials $(91.16 \pm 2 \%)$ than during LCS alone trials $(34.3 \pm 1 \%)$, and this difference was highly sig- 


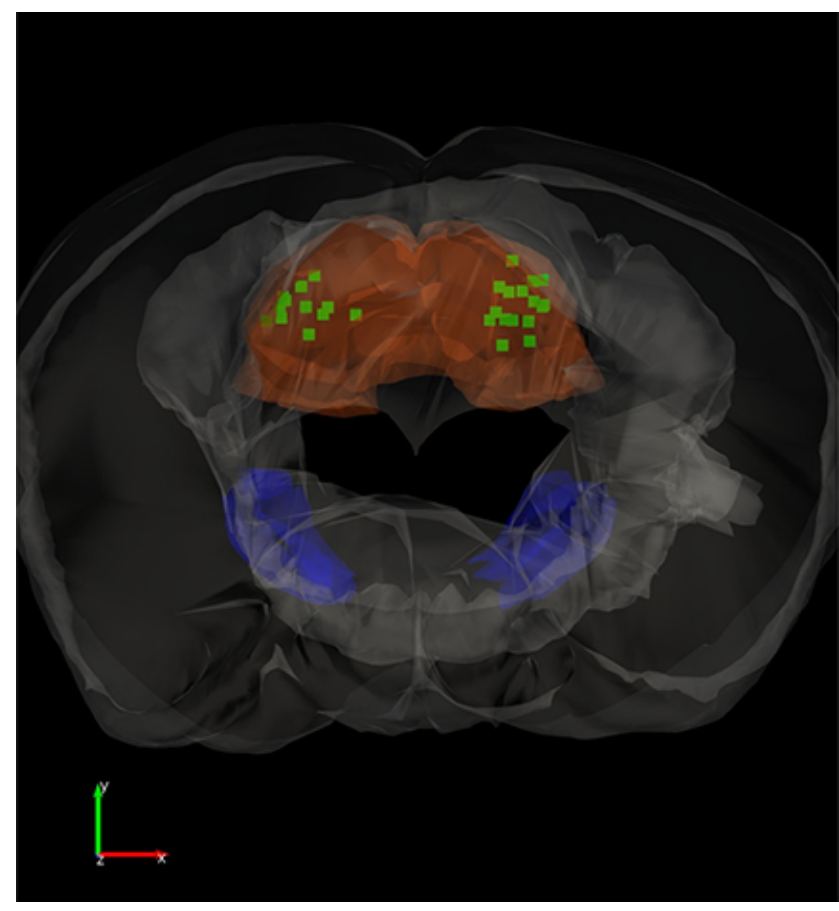

Movie 1. Video of the 3D reconstruction shown in Figure $2 B$.
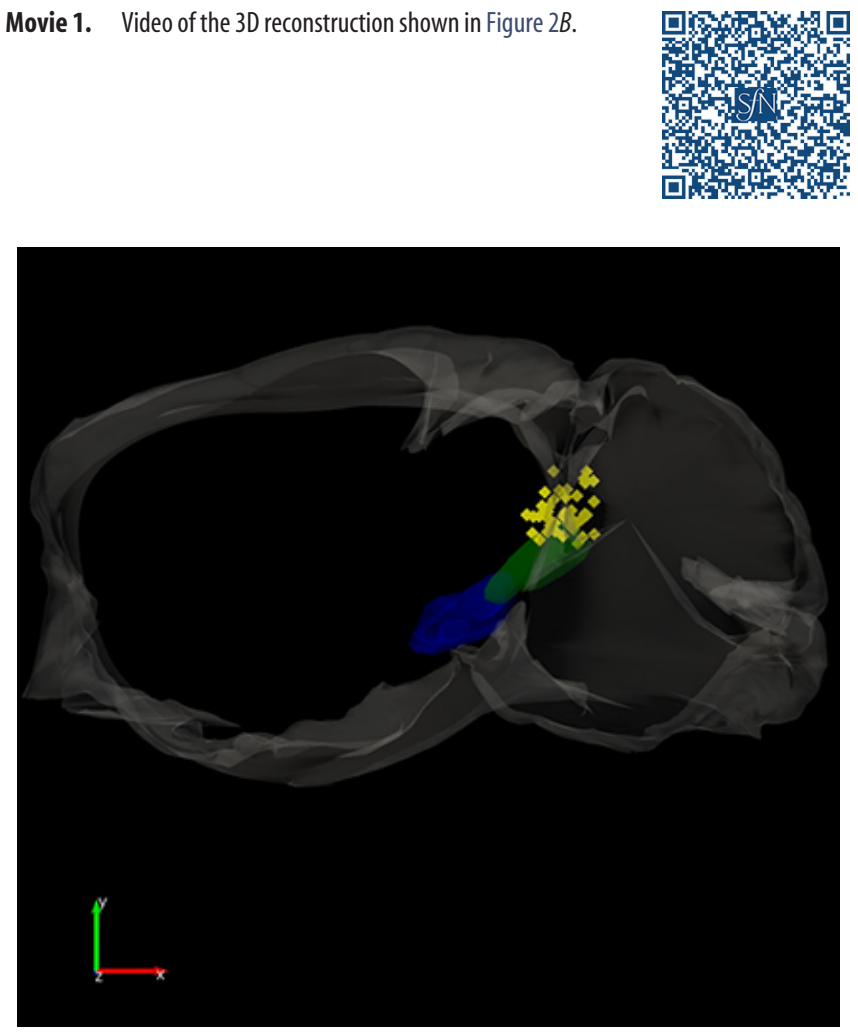

Movie 2. Video of the 3D reconstruction shown in Figure $2 C$.

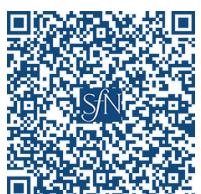

nificant (Tukey, $p<0.00001 ; 44$ sessions from 5 mice). The rate of avoidance in LCS alone trials for No Opsin animals is equivalent to that observed in No CS trials (Fig. $3 A$, C, open triangles vs red open circle); these responses occur by chance as a conse- quence of spontaneous movement and exploration. Thus, in ACS + LCS trials or LCS alone trials, the light per se in the absence of opsin expression, has no significant effect on the rate of avoidance responses. Furthermore, it is important to note that the effects we report are site-specific and/or light-pattern specific. Thus, they cannot be attributed to nonspecific effects of the light.

In conclusion, the results show that inhibitory inputs originating from SNr cells block avoidance responses in both the superior colliculus and PPT, but not in the thalamus. However, the suppressive effect of $\mathrm{SNr}$ output is much stronger in PPT than in the superior colliculus, requires less light power in PPT, and occurs for a broader range of optogenetic stimuli in PPT. In the superior colliculus, the suppression of avoidance responses occurs selectively for trains of blue light at $\sim 66 \mathrm{~Hz}$, but not for continuous light. In fact, continuous blue light in the superior colliculus has the opposite effect; it effectively drives avoidance responses (in the absence of an external CS).

\section{Intracellular postsynaptic correlates of GABAergic fiber excitation}

The preceding results show that activation of GABAergic fibers in a target nucleus with pulses of blue light delivered continuously or in trains at different frequencies produce contrasting effects on active avoidance performance. Using brain slices, we explored the intracellular postsynaptic correlates of these differences. Wholecell recordings were performed in the superior colliculus and PPT of Vgat-SNr-ChR2 mice (SNr-to-SC and SNr-to-PPT pathways). The stimulated GABAergic pathways maximally inhibited cell firing in the postsynaptic neuron when stimulated with trains of 1 ms pulses at $\sim 40 \mathrm{~Hz}$ (Fig. $5 A, B$ ), whereas continuous pulses were less effective. SNr GABAergic synapses inhibited superior colliculus $(n=13)$ cells somewhat more effectively with $40 \mathrm{~Hz}$ trains than with continuous pulses of blue light but this difference was not significant (Tukey, $p=0.38, p=0.58$, and $p=0.99 ; 40,66$, and $100 \mathrm{~Hz}$ vs Cont; Fig. 5B). In contrast, SNr GABAergic synapses inhibited PPT ( $n=15$ cells) cells more effectively with $40-100 \mathrm{~Hz}$ trains than with continuous pulses of blue light and this difference was significant (Tukey, $p=0.01, p=0.0004$, and $p=0.003 ; 40,66$ and $100 \mathrm{~Hz}$ vs Cont; Fig. $5 B$ ). To further peruse these differences in response dynamics, the area of hyperpolarization evoked during the initial $(0-100 \mathrm{~ms})$ and the final $(400-$ $500 \mathrm{~ms}$ ) portions of each light stimulus was measured to calculate the adaptation of the evoked IPSPs (Fig. 5C). Cells in the superior colliculus and PPT inhibited by SNr fibers showed similar levels of IPSP adaptation that were modest for trains up to $66 \mathrm{~Hz}$ and were maximal during continuous pulses (although adaptation was somewhat stronger in superior colliculus cells at the highest frequency trains; $66-100 \mathrm{~Hz}$ ). Many of the recorded cells were reconstructed. PPT cells had a multipolar shape with extensive dendrites oriented in the axis of the elongated PPT nucleus (Fig. $5 D$ ). Superior colliculus cells also had multipolar shapes but with more extensive dendritic arbors than PPT cells (Fig. 5E). A morphological analysis $(n=12 \mathrm{PPT}$ and $n=13$ superior colliculus cells) revealed that PPT cells tended to have larger somatic perimeters (Mann-Whitney, $U=114, p=0.026$ ), whereas superior colliculus cells tended to have higher dendritic complexity (MannWhitney, $U=42, p=0.026$; Neurolucida Explorer). In general, blue light trains at $40-66 \mathrm{~Hz}$ produce sustained IPSPs that are highly efficient at persistently inhibiting the postsynaptic neurons. In contrast, continuous pulses of blue light are associated with strong IPSP adaptation and weaker sustained inhibition. Interestingly, the same blue light applied in $\mathrm{SNr}$ to cells that express ChR2 produces cell firing that increases with train fre- 
A

B

$-\mathbf{-}-$ Vgat-SNr-ChR2 $\rightarrow$ VL $\quad-\mathrm{O}-$ Vgat-SNr-ChR2 $\rightarrow$ SC $\quad-\bullet-$ Vgat-SNr-ChR2 $\rightarrow$ PPT
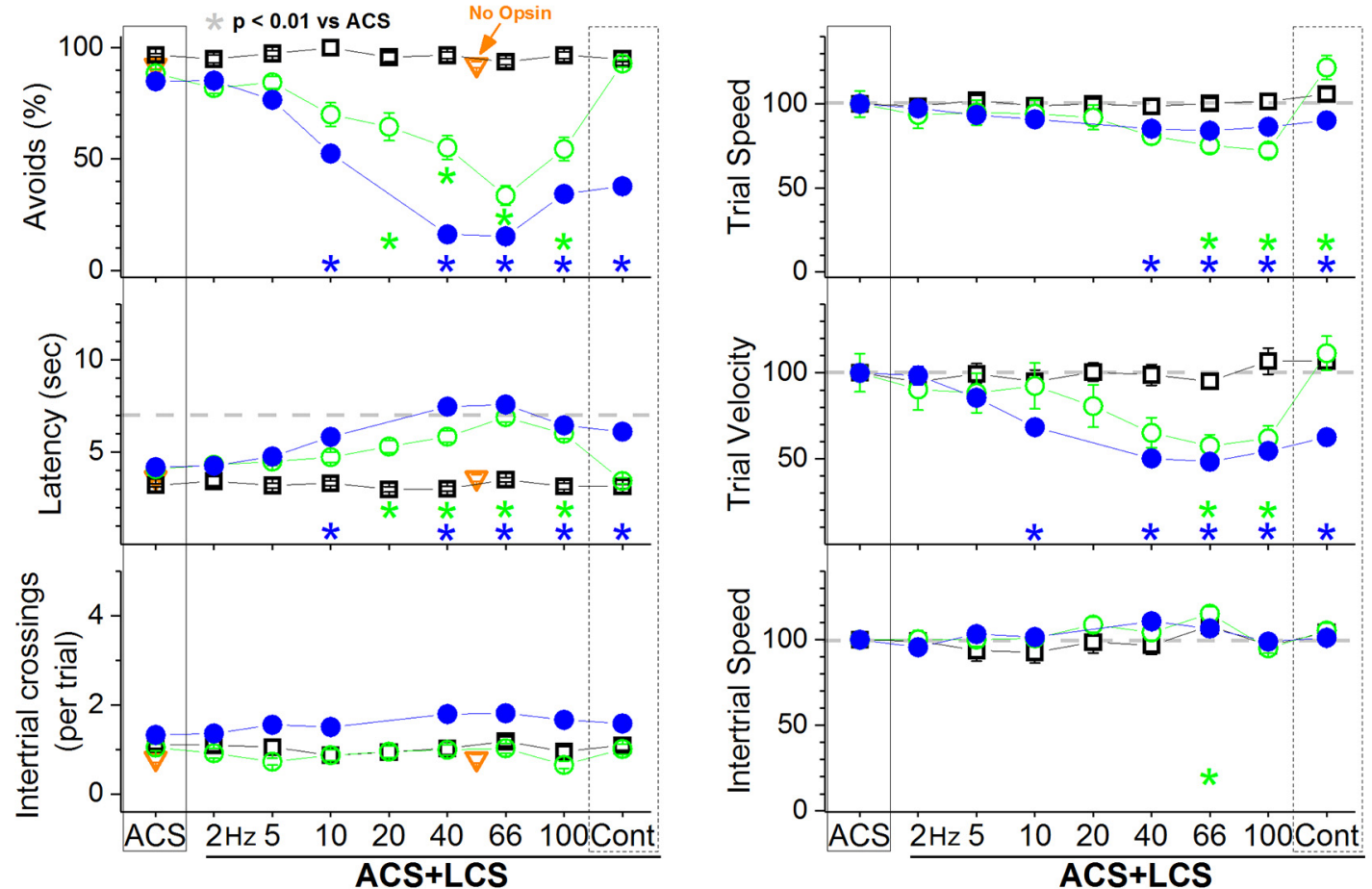

C

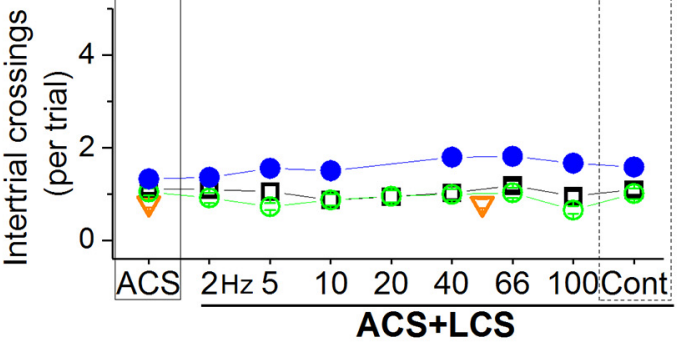

D

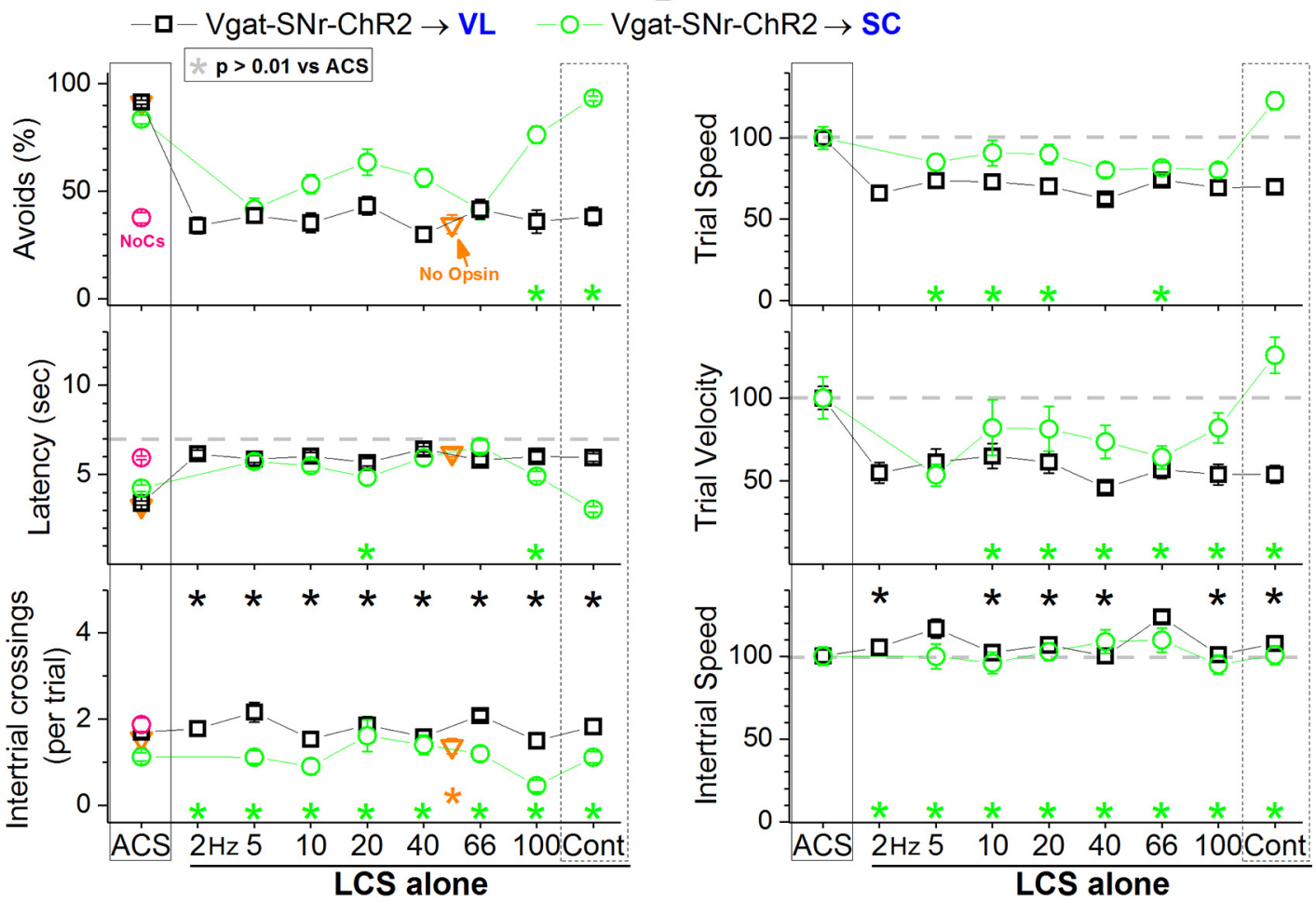

Figure 3. Effect of activating SNr output pathways on active avoidance responses. A, Effect of blue light applied in the ventrolateral/ventromedial (VL/VM) thalamus, superior colliculus, or PPT on ACS + LCS trials for animals that express ChR2 in GABAergic fibers originating in the SNr (Vgat-SNr-ChR2). ACS + LCS trials measure the effect of optogenetic stimulation on avoidance responses driven by the ACS. Plots in all figures display mean \pm SEM and asterisks denote Tukey tests. The plot also shows data for the No Opsin group of animals (open triangles), which compares the effect of all the light patterns used (combined together) versus ACS. B, Trial speed, trial velocity and intertrial speed for the data in $\boldsymbol{A}$. $C$, Effect of blue light applied in the VL/VM thalamus or superior colliculus on LCS alone trials for animals that express ChR2 in GABAergic fibers originating in the SNr (Vgat-SNr-ChR2). LCS alone trials measure the ability of the optogenetic stimulation to drive avoidance responses in the absence of the ACS. Note the efficacy of continuous blue light (cont) in the superior colliculus on driving avoidance responses. The asterisks highlight nonsignificant difference versus ACS trials. The plot also shows data for the No Opsin group of animals (open triangles). D, Trial speed, trial velocity, and intertrial speed for the data in $\boldsymbol{C}$. The asterisks highlight nonsignificant difference versus ACS trials. 


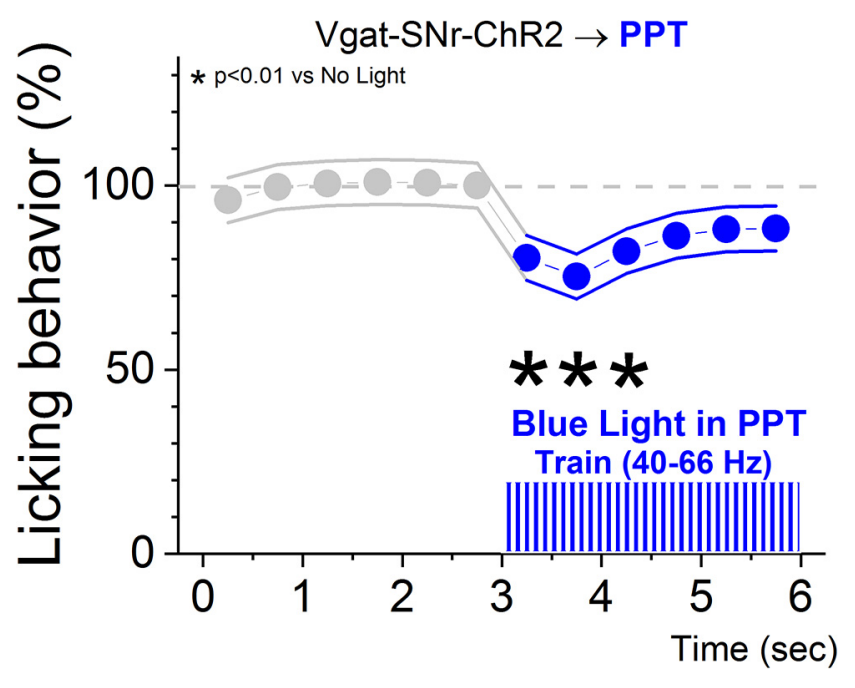

Figure 4. Effect of blue light in the PPT of Vgat-SNr-ChR2 mice on licking behavior. During the licking sessions, blue light was delivered ( $3 \mathrm{~s} 0 \mathrm{FF} / 3 \mathrm{~s}$ ON) in the PPT using trains of $1 \mathrm{~ms}$ pulses $(40-66 \mathrm{~Hz})$ at the same blue light power that blocked avoidance responses in each animal. The asterisks denote significant differences in licks between the light $0 \mathrm{~N}$ periods (six 0.5 s periods) and the last light $0 \mathrm{FF}$ period immediately preceding the light $0 \mathrm{~N}$ in the sequence.

quency, and continuous light produces the maximum cell firing (Hormigo et al., 2016). Therefore, when stimulating GABAergic fibers that express $\mathrm{ChR} 2$, the maximum postsynaptic inhibition is obtained with trains at $40-66 \mathrm{~Hz}$. But when stimulating cells (bodies and dendrites) that express ChR2, the maximum cell firing is obtained with continuous light.

We also conducted an additional set of experiments in urethane-anesthetized Vgat-SNr-ChR2 mice $(n=5)$ in which an optical fiber was placed in the superior colliculus and units were recorded (Fig. $5 F$ ) in the superior colliculus ( $n=31$ cells) and, in a few cases, simultaneously in PPT ( $n=6$ cells). As shown previously, in a different set of in vivo experiments (Hormigo et al., 2016), we found that the GABAergic synapses of SNr cells are very effective at inhibiting superior colliculus cells. Interestingly, blue light trains applied in the superior colliculus at $40 \mathrm{~Hz}$ also produced inhibitory responses in PPT cells that were recorded simultaneously. This indicates that activation of $\mathrm{SNr}$ fibers in the superior colliculus drives fiber collaterals of $\mathrm{SNr}$ cells that also project to PPT, because of antidromic activation of $\mathrm{SNr}$ cells. Such a finding is not surprising and is actually expected considering that individual $\mathrm{SNr}$ cells are known to project to multiple targets, including PPT and superior colliculus (Bentivoglio et al., 1979; Cebrián et al., 2005). Thus, excitation of SNr GABAergic fibers in superior colliculus inhibits both superior colliculus and PPT cells, and vice versa. Therefore, to clarify whether superior colliculus, PPT or both mediate active avoidance responses, in the following experiments, we independently modulated (excited or inhibited) the activity of superior colliculus and PPT (GABAergic or putative glutamatergic) cells during active avoidance. To target GABAergic cells we used Vgat-Cre mice. To target putative glutamatergic cells we used a CaMKII promoter. Note that while in some brain areas only glutamatergic cells express this enzyme, in other regions nonglutamatergic cells also express it (X. Wang et al., 2013). As shown in the following sections, the effects of manipulating the two targeted cell populations (Vgat and CaMKII) in our study were mostly opposite, indicating that we were targeting different cell groups.

\section{Inhibition of superior colliculus cells does not suppress} avoidance responses

To determine the effect of locally exciting or inhibiting GABAergic or putative glutamatergic cells in the superior colliculus on active avoidance, four groups of animals were implanted with bilateral optical fibers in the superior colliculus; optical fibers terminated in intermediate and deep layers (Fig. 2B). To excite GABAergic cells, a Cre-inducible AAV [AAV5-EF1a-DIOhChR2(H134R)-eYFP; UPenn Vector Core] was bilaterally injected in the superior colliculus of Vgat-Cre mice (Vgat-SCChR2). To inhibit GABAergic cells, Vgat-Arch mice (cross of Vgat-Cre and Ai40D mice to express ArchT in GABAergic cells) were used (Vgat-SC-Arch). To excite putative glutamatergic cells, an AAV with a CaMKII promoter [AAV5-CaMKIIa-hChR2 (H134R)-EYFP; UNC Vector Core] was bilaterally injected in the superior colliculus of C57BL/6J or Vgat-cre mice (CaMKII-SCChR2). To inhibit putative glutamatergic cells, an AAV with a CaMKII promoter (AAV5-CaMKIIa-eArchT3.0-EYFP; UNC Vector Core) was bilaterally injected in the superior colliculus of C57BL/6J or Vgat-Cre mice (CaMKII-SC-Arch). AAV injections resulted in EYFP expression that was localized within the superior colliculus (Fig. 2D).

In all these groups, we verified with whole-cell recordings in slices that the opsins (AAVs) were working as expected in superior colliculus and/or PPT. For instance, we selected eArchT3.0 (Chow et al., 2010; Mattis et al., 2011) for our experiments because compared with ArchT, which we also tested previously in slices (Hormigo et al., 2016), cells expressing eArchT3.0 ( $n=15$ cells) produced notably stronger membrane hyperpolarization in response to green light (data not shown; but see Mattis et al., 2011). This completely abolished all spontaneous firing (like with ArchT), but also all evoked firing (triggered by injected positive current pulses) up to the highest firing rates we could evoke in these cells. In addition, these optogenetics experiments were supplemented with DREADDs experiments to excite or inhibit superior colliculus cells using AAV's that we tested previously (Hormigo et al., 2016).

Inhibition of GABAergic cells in the superior colliculus with continuous green light improved the ability to avoid the US in ACS+LCS trials (Fig. 6B, black closed circles, Vgat-SC-Arch; 36 sessions in 4 mice). There was a small increase in the percentage of avoidance responses (Tukey, $p=0.016$ ) and a decrease in latency $(p<0.00001)$ compared with ACS trials. Video tracking showed (Fig. $6 C$ ) an increase in both trial speed $(p=0.0003)$ and trial velocity ( $p=0.0079$ ) compared with ACS trials, indicating that the animals avoided more rapidly when GABAergic cells in superior colliculus were inhibited. Furthermore, inhibition of GABAergic cells in superior colliculus cells with DREADDs did not affect avoidance responses driven by the ACS (Fig. 7A, black filled circles, Vgat-SC-hM4Di; 60 sessions in 5 mice), which emphasizes the importance of inhibiting these cells selectively during the CS period (avoidance interval) to facilitate avoidance responses.

Inhibition of putative glutamatergic cells in the superior colliculus with continuous green light did not affect the ability to avoid the US in ACS+LCS trials (Fig. 6B, red closed squares, CaMKII-SC-Arch; 21 sessions in 5 mice). Video tracking did not reveal (Fig. 6C) effects on movement during the task. In these animals (and in the equivalent animals shown below targeting PPT; CaMKII-PPT-Arch), the green light was turned off slowly (in a few seconds) using a linearly decaying pulse during the onset of the intertrial interval, because abruptly turning off the light caused behavioral reactions (during the intertrial interval) typical 

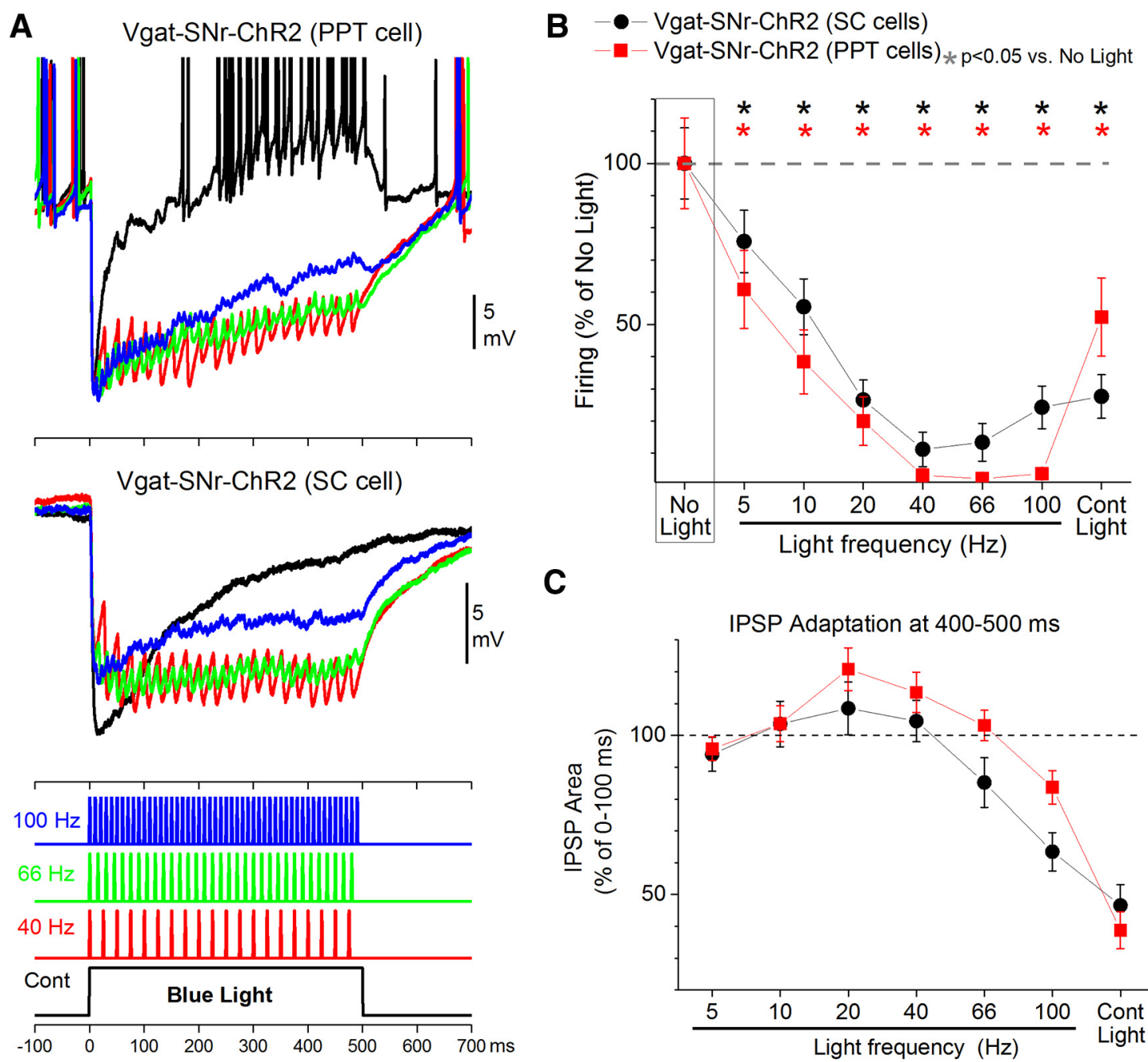

C
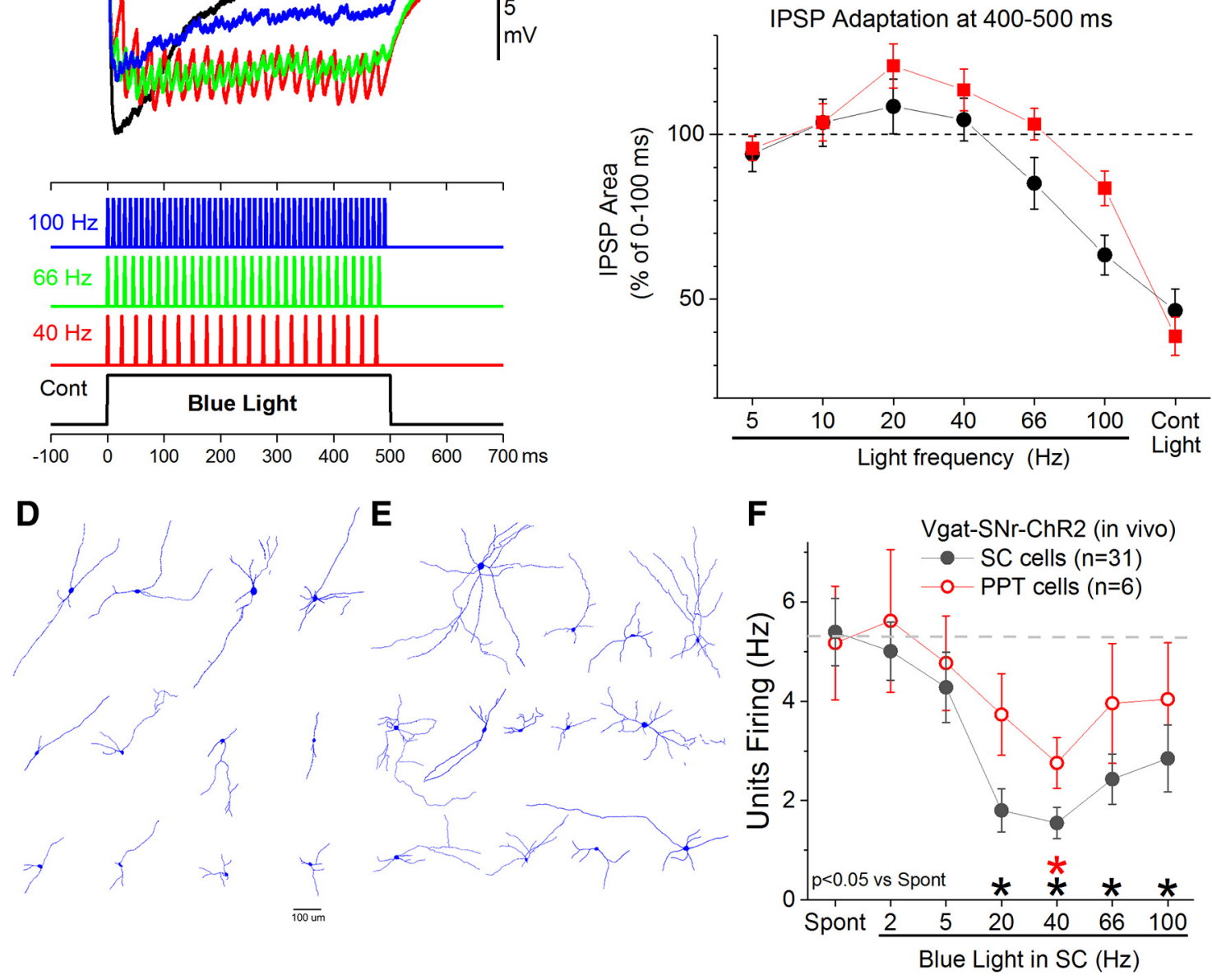

Figure 5. Postsynaptic effects of activating GABAergic fibers with optogenetics. A, Example intracellular whole-cell responses obtained in two different neurons after activating GABAergic fibers that express ChR2 with continuous pulses or trains of 1 ms pulses of blue light. The GABAergic fibers originate in SNr and the recorded cells are in PPT (top) or superior colliculus (bottom). Each trace is an average of six trials. $B$, Effect of blue light on the firing of neurons recorded intracellularly for the different pathways: SNr-to-PPT and SNr-to- superior colliculus (SC). Firing was spontaneous (resting $V_{m}$ ) or induced with positive current injection. C, Adaptation of the hyperpolarizing responses (IPSPS) caused by activating GABAergic fibers. The area of hyperpolarization measured at the end $(400-500 \mathrm{~ms}$ ) of each type of optogenetic stimulus is plotted as a percentage of the area at the onset ( $0-100 \mathrm{~ms})$ of the stimulus to reveal the amount of adaptation of the response as a function of the stimulus type. $\boldsymbol{D}$, Cells $(n=12)$ recorded in PPT that were inhibited by SNr GABAergic afferents. $\boldsymbol{E}$, Cells $(n=13)$ recorded in superior colliculus that were inhibited by SNr GABAergic afferents. $\boldsymbol{F}$, Units recorded in the superior colliculus and PPT of urethane-anesthetized Vgat-SNr-Chr2 mice during application of blue light trains in the superior colliculus. Blue light trains delivered in superior colliculus at $40 \mathrm{~Hz}$ inhibit cells in PPT, presumably through antidromic activation of SNr cells that project to both superior colliculus and PPT. 

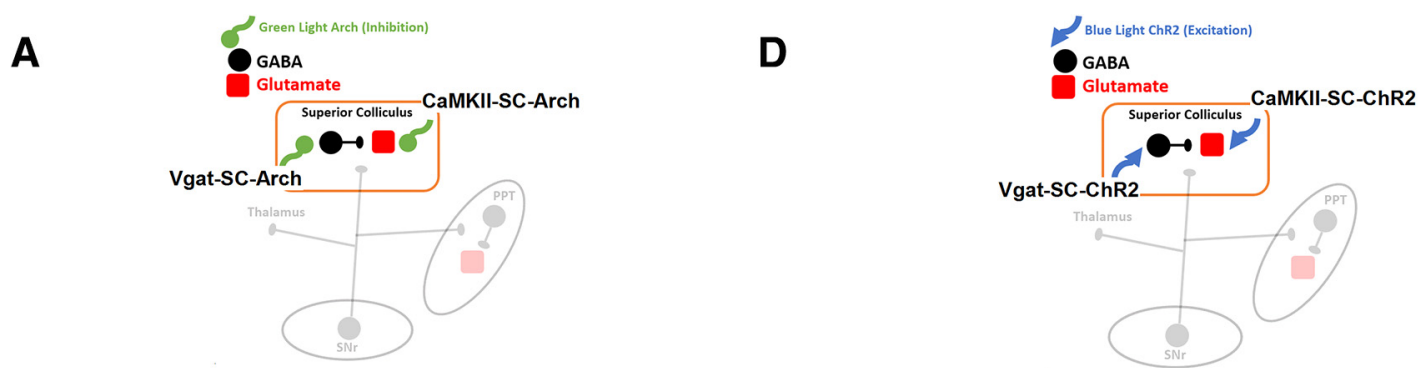

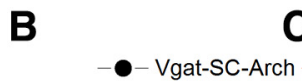

C
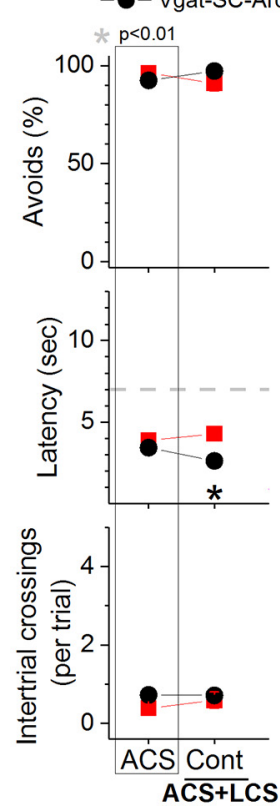

- CaMKII-SC-Arch
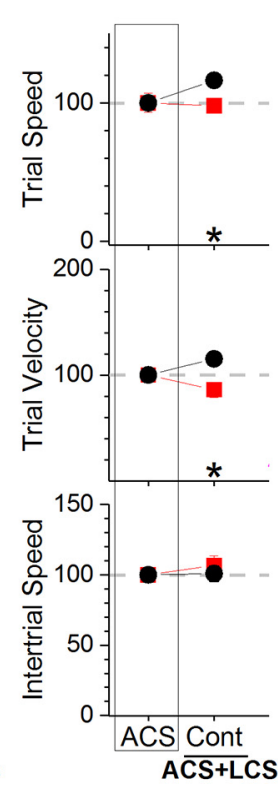

E

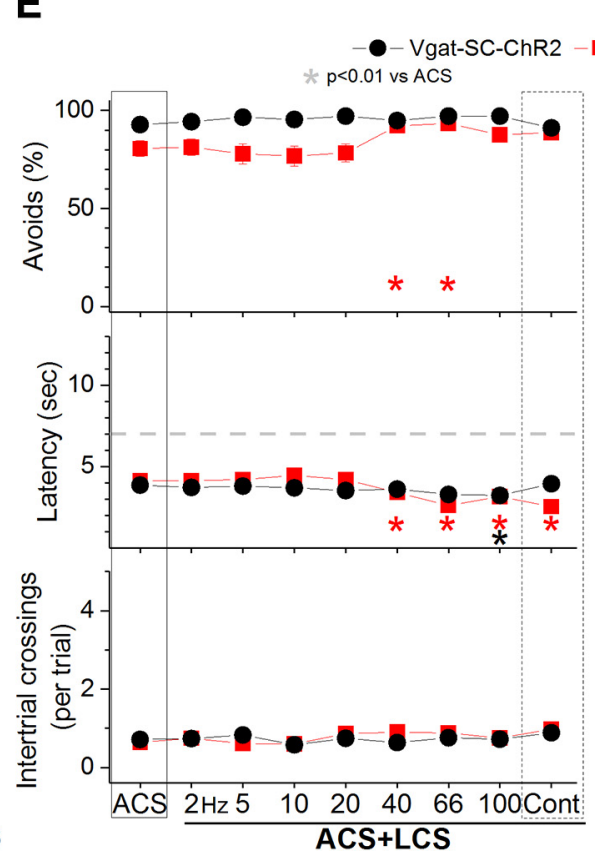

$\mathbf{F}$

- CaMKII-SC-ChR2
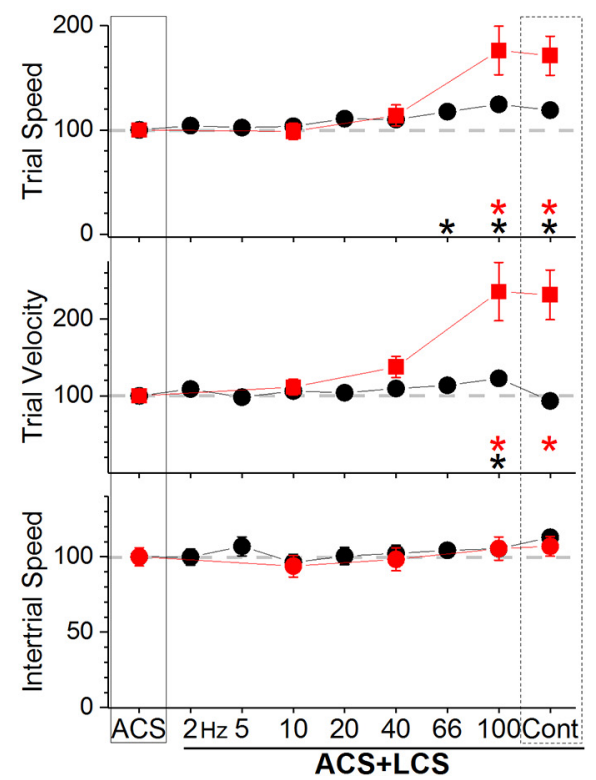

Figure 6. Effect of activating or inhibiting cells in the superior colliculus on avoidance responses driven by the ACS. $A$, Schematic of the superior colliculus cells inhibited in the experiments shown in $\boldsymbol{B}$ and $\boldsymbol{C}$. $\boldsymbol{B}$, Effect of green light applied in the superior colliculus on ACS + LCS trials for animals that express Arch in GABAergic (Vgat-SC-Arch) or putative glutamatergic (CaMKII-SC-Arch) cells in the superior colliculus. $\boldsymbol{C}$, Trial speed, trial velocity, and intertrial speed for the data in $\boldsymbol{B}$. D, Schematic of the superior colliculus cells activated in the experiments shown in $\boldsymbol{E}$ and $\boldsymbol{F}$. $\boldsymbol{E}$, Effect of blue light applied in the superior colliculus on ACS + LCS trials for animals that express ChR2 in GABAergic (Vgat-SC-ChR2) or putative glutamatergic (CaMKII-SC-ChR2) cells in the superior colliculus. $\boldsymbol{F}$, Trial speed, trial velocit,y and intertrial speed for the data in $\boldsymbol{B}$.

of exciting these cells (e.g., escaping). These reactions are because of rebounds of neuronal firing produced by the strong hyperpolarization induced by the more potent variants (eArchT3.0) of Arch (Wiegert et al., 2017). The slow ramping of the light after the trial ended completely eliminated the behavioral reactions. Similar to the optogenetics effects, inhibition of superior colliculus cells (targeted with a hSyn promoter) with DREADDs did not affect avoidance responses driven by an ACS (Fig. 7A, hSyn-SChM4Di; 96 sessions in 8 mice). The fact that inhibiting superior colliculus cells with various manipulations does not affect avoidance responses driven by the ACS supports the contention that the suppression of avoidance responses caused by exciting $\mathrm{SNr}$ fibers in the superior colliculus at $\sim 66 \mathrm{~Hz}$ (Fig. $3 A$, green open circles) is not because of the inhibition of superior colliculus cells.

In LCS alone trials (Fig. 8B), inhibition of either GABAergic (Fig. $8 B$, black closed circles, Vgat-SC-Arch; 31 sessions in 5 mice) or putative glutamatergic cells (Fig. $8 B$, red closed squares, CaMKII-SC-Arch; 29 sessions in 4 mice) in the superior colliculus functioned as a very effective CS to drive avoidance responses in the absence of an external ACS. The percentage of avoidance responses in LCS alone trials was much higher than in No CS trials. Thus, superior colliculus inhibition is an effective CS to drive avoidance responses. This agrees with the finding (Fig. $3 C$, green open circles) that inhibiting superior colliculus cells by stimulating $\mathrm{SNr}$ fibers with continuous pulses drives avoidance responses.

\section{Excitation of superior colliculus cells drives} avoidance responses

Excitation of GABAergic cells in the superior colliculus with blue light $(2.3 \mathrm{~mW}$, range: $1.8-2.8)$ had little effect on the ability to avoid the US in ACS+LCS trials (Fig. 6E, black closed circles, Vgat-SC-ChR2; 35 sessions in 7 mice). This was the case even after increasing the light power to a high level (percentage of avoidances: Tukey, $p>0.6$, for $2-100 \mathrm{~Hz}$ and Cont vs ACS; 20 sessions in 3 mice). However, at the regular light power, video tracking revealed (Fig. $6 F$ ) an increase in both trial speed (Tukey, $p<0.00001 ; 66,100 \mathrm{~Hz}$ and Cont vs ACS) and trial velocity ( $p=$ $0.0002 ; 100 \mathrm{~Hz}$ vs ACS) for blue light $\sim 100 \mathrm{~Hz}$, which also showed a faster latency (Fig. $6 E$, middle; $p=0.01$ and $p=0.0055$; 66 and $100 \mathrm{~Hz}$ vs ACS). Excitation of GABAergic cells in superior colliculus with DREADDs had a small suppressive effect (11.6\%; $p<0.00001$ ) on the percentage of avoidance responses driven by the ACS and increased latency $(p=0.0002)$ by $0.82 \mathrm{~s}$ (Fig. $7 B$, Vgat-SC-hM3Dq; 60 sessions in 5 mice). The inability of the DREADDs excitation of these cells to drive avoidance responses emphasizes the importance of exciting GABAergic superior col- 
A

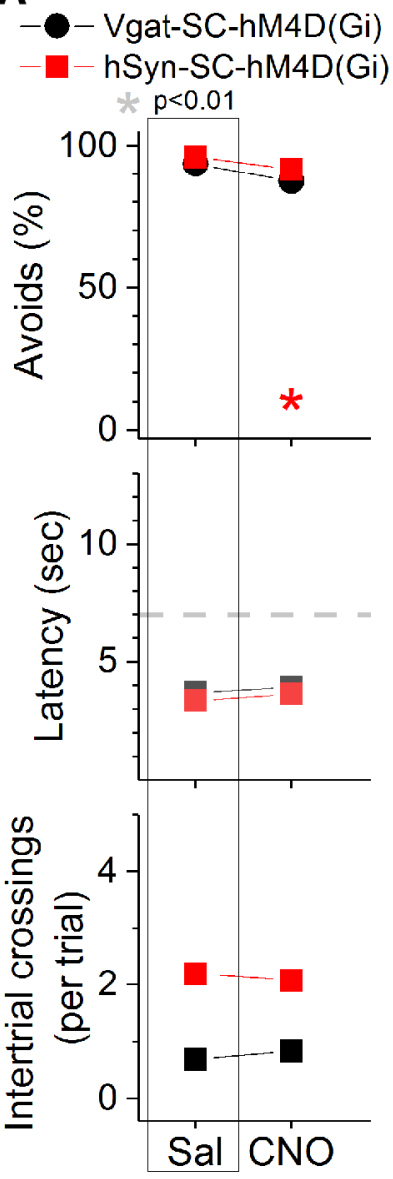

B

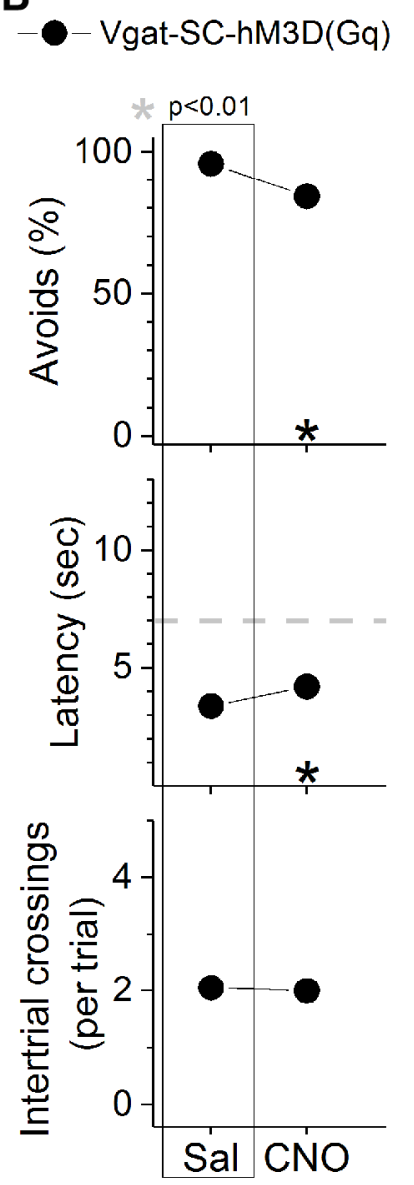

C
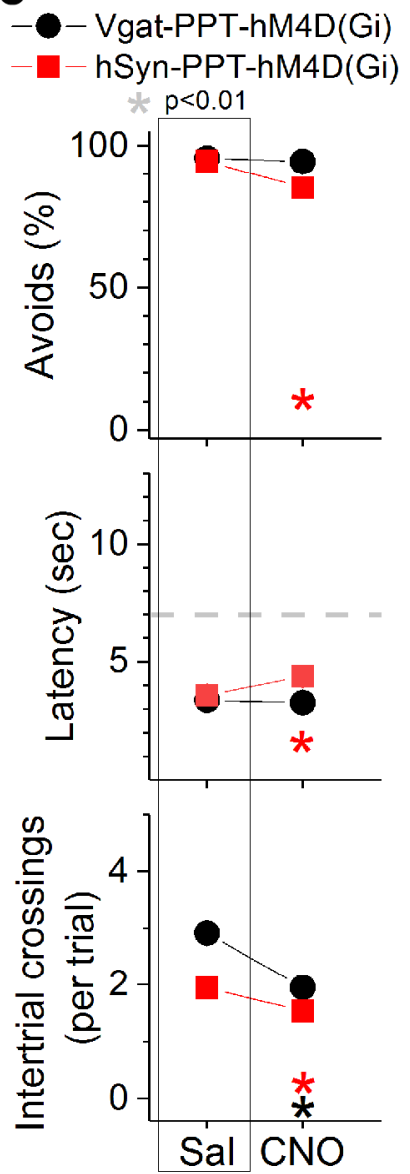

D
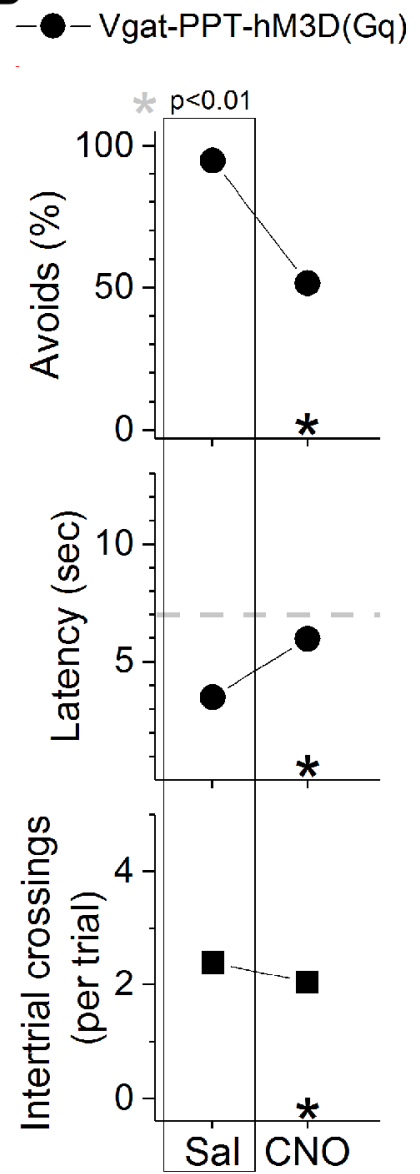

Figure 7. Effect of DREADDs in superior colliculus or PPT on active avoidance. Comparison of the effects of saline and CNO on ACS trials in mice that express hM4D(Gi) or hM3D(Gq) in GABAergic neurons (black circles) or non-selectively in neurons (hSyn promoter; red squares) of superior colliculus $(\boldsymbol{A}, \boldsymbol{B})$ or PPT $(\boldsymbol{C}, \boldsymbol{D})$.

liculus cells selectively during the avoidance interval to drive avoidance responses.

Excitation of putative glutamatergic cells in superior colliculus with blue light (1.4 $\mathrm{mW}$, range: $0.4-2.8)$ had a strong facilitating effect on the ability to avoid the US in ACS + LCS trials (Fig. 6E, red closed squares, CaMKII-SC-ChR2; 37 sessions in 6 mice). The percentage of avoids increased for blue light stimuli of $40-66 \mathrm{~Hz}(p=0.0029$ and $p=0.0008 ; 40$ and 66 $\mathrm{Hz}$ vs ACS), and the latency decreased for stimuli $>20 \mathrm{~Hz}$, including continuous blue light $(p=0.0002, p<0.00001, p<0.00001$, and $p<0.00001 ; 40,66,100 \mathrm{~Hz}$ and Cont vs ACS). Video tracking revealed (Fig. $6 F$ ) a strong increase in trial speed, and especially trial velocity, for $100 \mathrm{~Hz}$ trains $(p<0.00001)$ and continuous $(p<$ $0.00001)$ blue light stimuli.

In LCS alone trials (Fig. 8E), excitation of either GABAergic (Fig. 8E, black closed circles, Vgat-SC-ChR2; 73 sessions in 7 mice) or putative glutamatergic (Fig. $8 E$, red closed squares, CaMKII-SC-ChR2; 64 sessions in 6 mice) cells in the superior colliculus were both very effective CS to drive avoidance responses in the absence of an external ACS. The ability to drive avoidance responses was more selective when GABAergic cells were excited; this was effective for light trains $>10 \mathrm{~Hz}$, including continuous light $(p=0.9, p=0.99, p=0.99, p=0.56, p=0.99$; $20,40,66,100 \mathrm{~Hz}$ and Cont vs ACS). Exciting putative glutamatergic cells was also effective even with blue light train frequencies as low as $2 \mathrm{~Hz}$. Video tracking revealed (Fig. $8 F$ ) that trial speed and trial velocity were either equivalent or faster compared with ACS trials.

In conclusion, superior colliculus cells are not essential for active avoidance responses driven by an ACS, but modulation of these cells (inhibition and, especially, excitation) serves as a very effective CS to drive avoidance responses in the absence of an external CS.

\section{Inhibition of putative glutamatergic PPT cells blocks avoidance responses}

To inhibit or excite GABAergic and putative glutamatergic cells in PPT we used the same strategies (groups) as in the superior colliculus (see previous two sections) except that AAV injections targeted the PPT (Fig. 2C). AAV injections resulted in EYFP expression in PPT but also in some immediately adjacent areas, which varied between mice (Fig. $2 E$ ). Thus, the common feature among the animals was EYFP expression in PPT and an optical fiber localized in the middle to dorsal portion of PPT. As done in the superior colliculus, four groups of animals were implanted with bilateral optical fibers in PPT (Vgat-PPT-Arch, CaMKII-PPTArch, Vgat-PPT-ChR2, and CaMKII-PPT-ChR2), and DREADDs experiments were also conducted to excite or inhibit PPT cells during active avoidance (Fig. $7 C, D$ ).

Inhibition of GABAergic cells in the PPT with continuous green light improved the ability to avoid the US in ACS+LCS trials (Fig. 9B, black closed circles, Vgat-PPT-Arch; 23 sessions in 

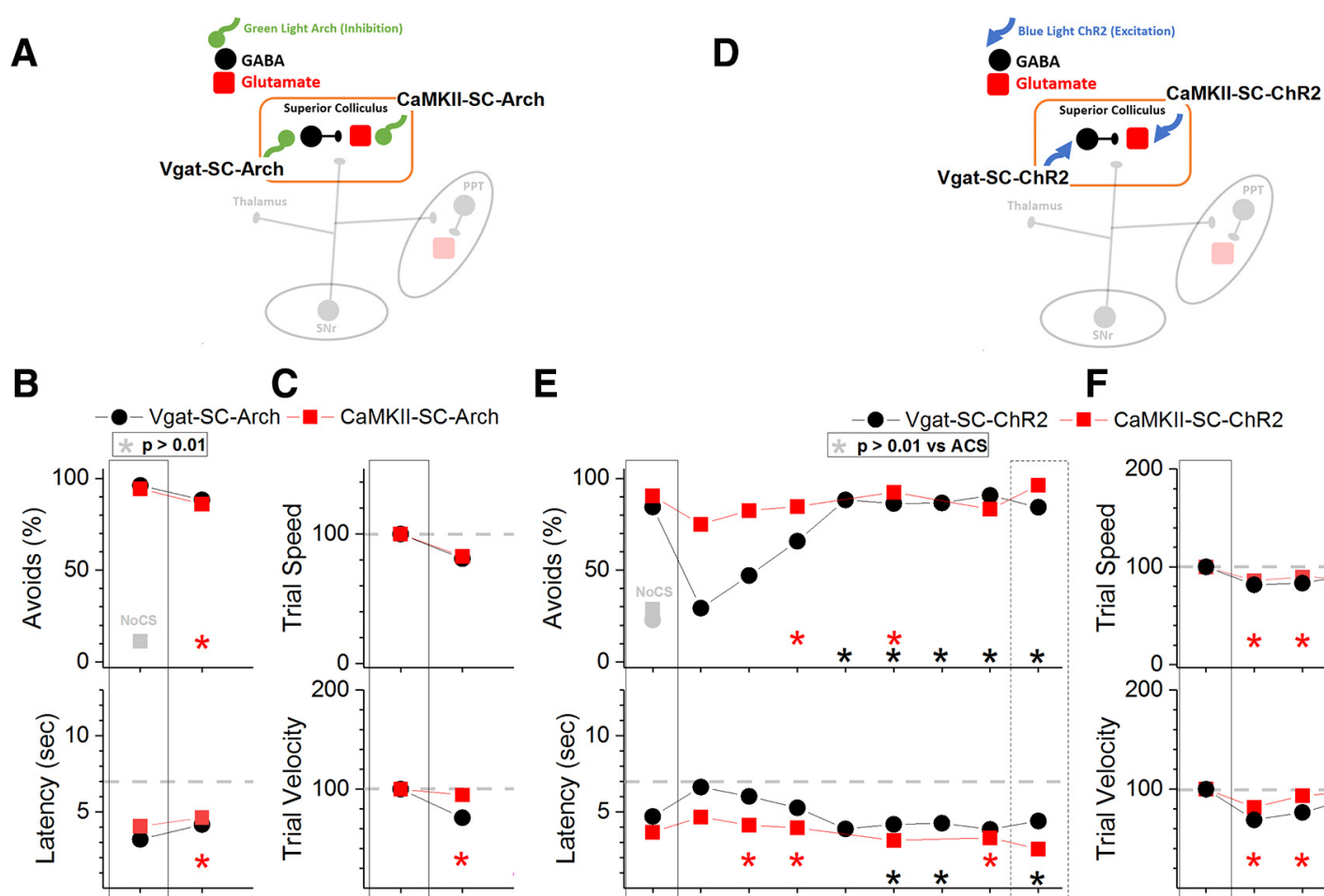

C
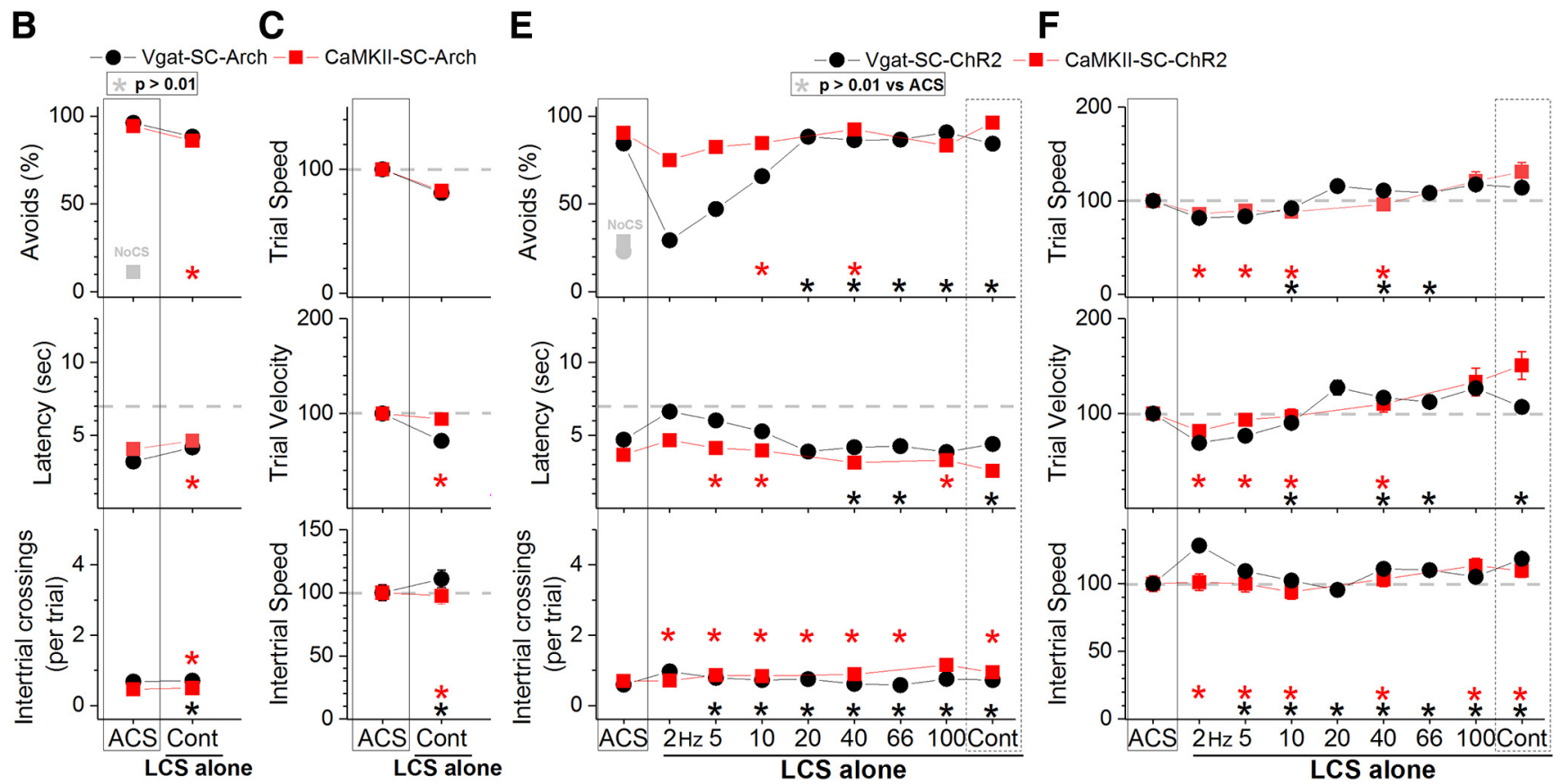

Figure 8. Effect of activating or inhibiting cells in the superior colliculus on driving avoidance responses in the absence of the ACS. A, Schematic of the superior colliculus cells inhibited in the experiments shown in $\boldsymbol{B}$ and $\boldsymbol{C} . \boldsymbol{B}$, Effect of green light applied in the superior colliculus on LCS alone trials for animals that express Arch in GABAergic (Vgat-SC-Arch) or putative glutamatergic (CaMKII-SC-Arch) cells in the superior colliculus. The asterisks highlight nonsignificant difference versus ACS trials. C, Trial speed, trial velocity, and intertrial speed for the data in $\boldsymbol{B}$. The asterisks highlight nonsignificant difference versus ACS trials. $\boldsymbol{D}$, Schematic of the superior colliculus cells activated in the experiments shown in $\boldsymbol{E}$ and $\boldsymbol{F}$. $\boldsymbol{E}$, Effect of blue light applied in the superior colliculus on LCS alone trials for animals that express ChR2 in GABAergic (Vgat-SC-ChR2) or putative glutamatergic (CaMKII-SC-ChR2) cells in the superior colliculus. The asterisks highlight nonsignificant difference versus ACS trials. $\boldsymbol{F}$, Trial speed, trial velocity, and intertrial speed for the data in $\boldsymbol{E}$. The asterisks highlight nonsignificant difference versus ACS trials.

4 mice). There was an increase in the percentage of avoidance responses (Tukey, $p=0.0005)$ and a decrease in latency $(p<$ $0.00001)$ compared with ACS trials. Video tracking showed (Fig. $9 C)$ an increase of both trial speed $(p=0.00027)$ and velocity $(p=0.0003)$ compared with ACS trials, indicating that the animals avoided more rapidly when GABAergic cells in PPT were inhibited. Similarly, in LCS alone trials (Fig. 10B), inhibition of GABAergic cells in PPT was a very effective CS to drive avoidance responses (Fig. 10B, black closed circles, Vgat-PPT-Arch; 21 sessions in 4 mice). Inhibition of GABAergic cells in PPT with DREADDs had no effect on avoidance responses driven by an ACS (Fig. 7C, Vgat-PPT-hM4Di; 84 sessions in 7 mice), indicating that suppression of these cells unrelated to the avoidance interval is not effective at driving avoidance responses.

Inhibition of putative glutamatergic cells in PPT with continuous green light abolished the ability to avoid the US in ACS + LCS trials (Fig. 9B, red closed squares, CaMKII-PPT-Arch; 27 sessions in 5 mice). Both the percentage of avoids $(p<$ $0.00001)$ and the latency $(p<0.00001)$ were strongly affected. Video tracking showed (Fig. $9 C$ ) a suppression of both trial speed $(p<0.00001)$ and velocity $(p<0.00001)$ indicating that movement was suppressed during inhibition of putative glutamatergic cells in PPT. Importantly, although the animals did not avoid in response to the ACS they readily escaped in response to the US, displaying fast escape latencies at US onset. The effects of different green light power levels were compared within the same sessions in these animals, which revealed that the animals escaped even at the highest power levels used (Fig. 11A). Considering the strong suppression of avoidance responses, these animals were not subjected to LCS alone trials. Inhibition of PPT neurons with DREADDs produced a small $(9.4 \%)$ suppression of the percentage of avoidance responses $(p=0.00007)$ and an increase in latency ( $p=0.0004)$ of $0.79 \mathrm{~s}$ (Fig. 7C, hSyn-PPT-hM4Di; 72 sessions in 6 mice). The much stronger effect of optogenetics compared with DREADDs is likely because of much stronger inhibition of neural activity caused by optogenetics, particularly with the new generation eArchT3.0 we used; DREADDs only partially inhibits cell firing in vivo (Hormigo et al., 2016).

In conclusion, inhibition of GABAergic cells in PPT is an effective driver of avoidance responses. Inhibition of putative glutamatergic cells in PPT abolishes active avoidance responses to the ACS while preserving escape responses to the US. Putative glutamatergic cells in PPT are necessary for active avoidance responses. 

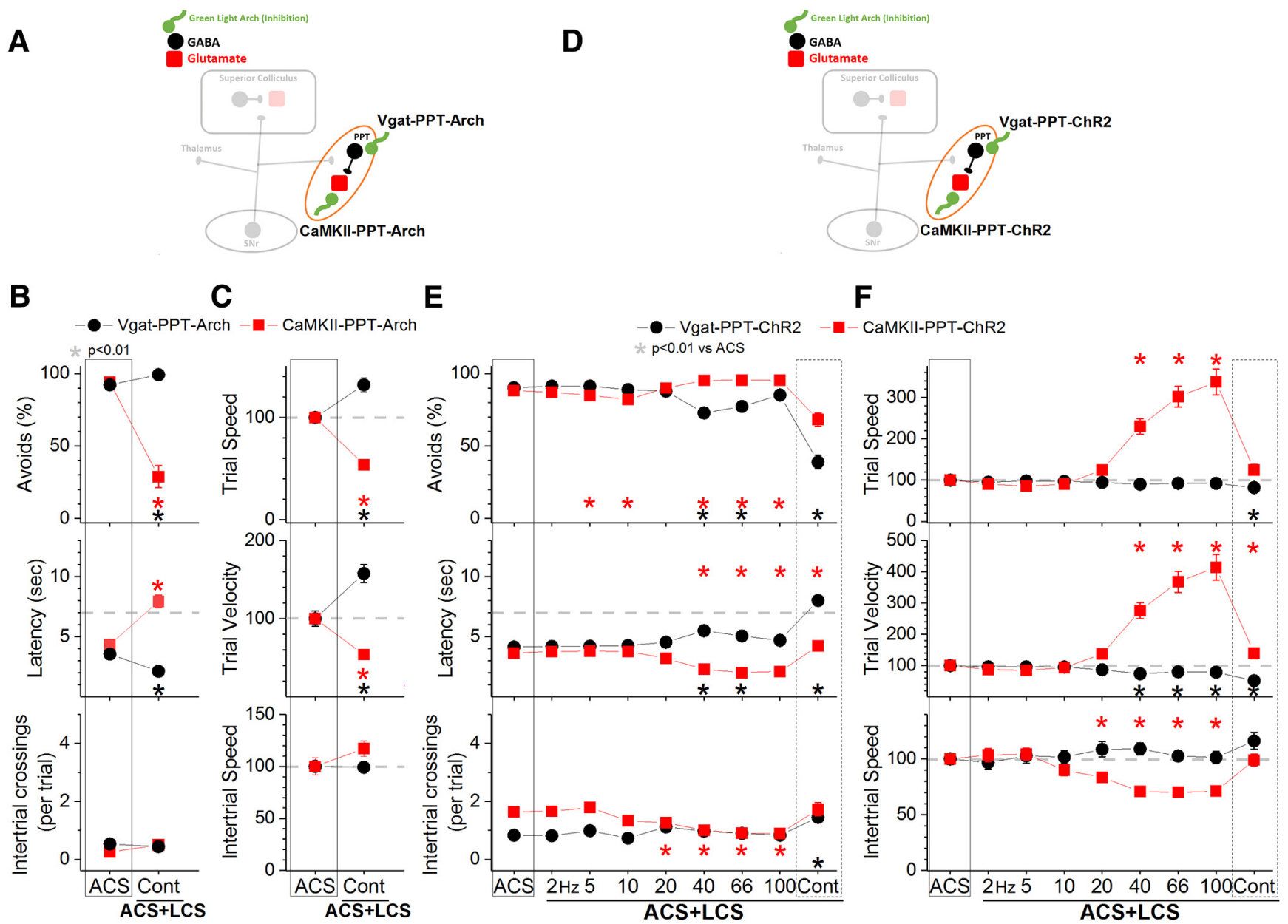

Figure 9. Effect of activating or inhibiting cells in the PPT on avoidance responses driven by the ACS. $\boldsymbol{A}$, Schematic of the PPT cells inhibited in the experiments shown in $\boldsymbol{B}$ and $\boldsymbol{C}$. $\boldsymbol{B}$, Effect of green light applied in the PPT on ACS + LCS trials for animals that express Arch in GABAergic (Vgat-PPT-Arch) or putative glutamatergic (CaMKII-PPT-Arch) cells in PPT. C, Trial speed, trial velocity, and intertrial speed for the data in B. D, Schematic of the PPT cells activated in the experiments shown in $\boldsymbol{E}$ and $\boldsymbol{F}$. $\boldsymbol{E}$, Effect of blue light applied in the PPT on ACS $+\mathrm{LCS}$ trials for animals that express $C$ hR2 in GABAergic (Vgat-PPT-ChR2) or putative glutamatergic (CaMKII-PPT-ChR2) cells in the PPT. $\boldsymbol{F}$, Trial speed, trial velocity, and intertrial speed for the data in $\boldsymbol{E}$.

\section{Excitation of GABAergic PPT cells blocks avoidance responses}

Excitation of GABAergic cells in PPT with blue light $(1.7 \mathrm{~mW}$, range: 0.3-3.4) suppressed the ability to avoid the US in ACS + LCS trials (Fig. 9E, black closed circles, Vgat-PPT-ChR2; 46 sessions in 7 mice). The effect on the percentage of avoidance responses and on latency was strong for continuous pulses of blue light $(p<0.00001)$, but much weaker for trains at $40-66 \mathrm{~Hz}$ $(p=0.00001$ and $p=0.01 ; 40$ and $66 \mathrm{~Hz}$ vs ACS). Video tracking revealed (Fig. $9 F$ ) a decrease in trial speed and velocity for continuous pulses of blue light $(p<0.00001)$, and in trial velocity for trains of light at $40-100 \mathrm{~Hz}(p<0.00001, p=0.0002, p=$ $0.00016 ; 40,66$, and $100 \mathrm{~Hz}$ vs ACS). Thus, similar to the inhibition of putative glutamatergic cells, the excitation of GABAergic cells in PPT suppressed active avoidance and also movement, particularly when continuous blue light was used. Interestingly, slightly increasing the blue light power in a subgroup of these animals $(n=3)$ began to suppress escape responses by delaying the response latency during continuous pulses (Fig. 11B). This contrasts with the effect of increasing the green light power level to inhibit putative glutamatergic cells in PPT, which as noted above did not increase the latency of escape responses. The finding that exciting GABAergic cells in PPT suppresses avoidance responses was supported by DREADDs experiments, which showed a strong suppression (43.3\%) of the percent- age of avoidance responses $(p<0.00001)$ driven by the ACS, an increase in latency $(2.5 s ; p<0.00001)$ and a slight suppression in intertrial crossings ( $p=0.007$ ) when GABAergic cells in PPT were excited (Fig. 7D, Vgat-PPT-hM3Dq; 60 sessions in 5 mice). In conclusion, excitation of GABAergic cells in PPT blocks avoidance responses.

\section{Excitation of putative glutamatergic PPT cells drives} avoidance and faster escape responses

Excitation of putative glutamatergic cells in PPT with blue light (1.8 mW, range: 0.4-3.4) in ACS + LCS trials (Fig. 9E, red closed squares, CaMKII-PPT-ChR2; 76 sessions in 7 mice) significantly increased the percentage of avoidance responses for blue light stimuli $>20 \mathrm{~Hz}$ including continuous light $(p=0.00056, p=$ $0.00079, p=0.00062$, and $p<0.00001 ; 40,66,100$ and Cont vs ACS), and reduced the latency $(p<0.00001)$. Avoidance responses increased in speed as a function of the blue light train frequency. Increasing the blue light frequency $>20 \mathrm{~Hz}$ lead to strong increases in speed so that avoidance responses at the highest frequencies resembled escape responses. At $100 \mathrm{~Hz}$, avoidance responses were up to 3-5 times faster than avoidance responses in ACS trials.

Similar effects were observed in LCS alone trials (Fig. 10E, red closed squares, CaMKII-PPT-ChR2; 36 sessions in 7 mice). With 

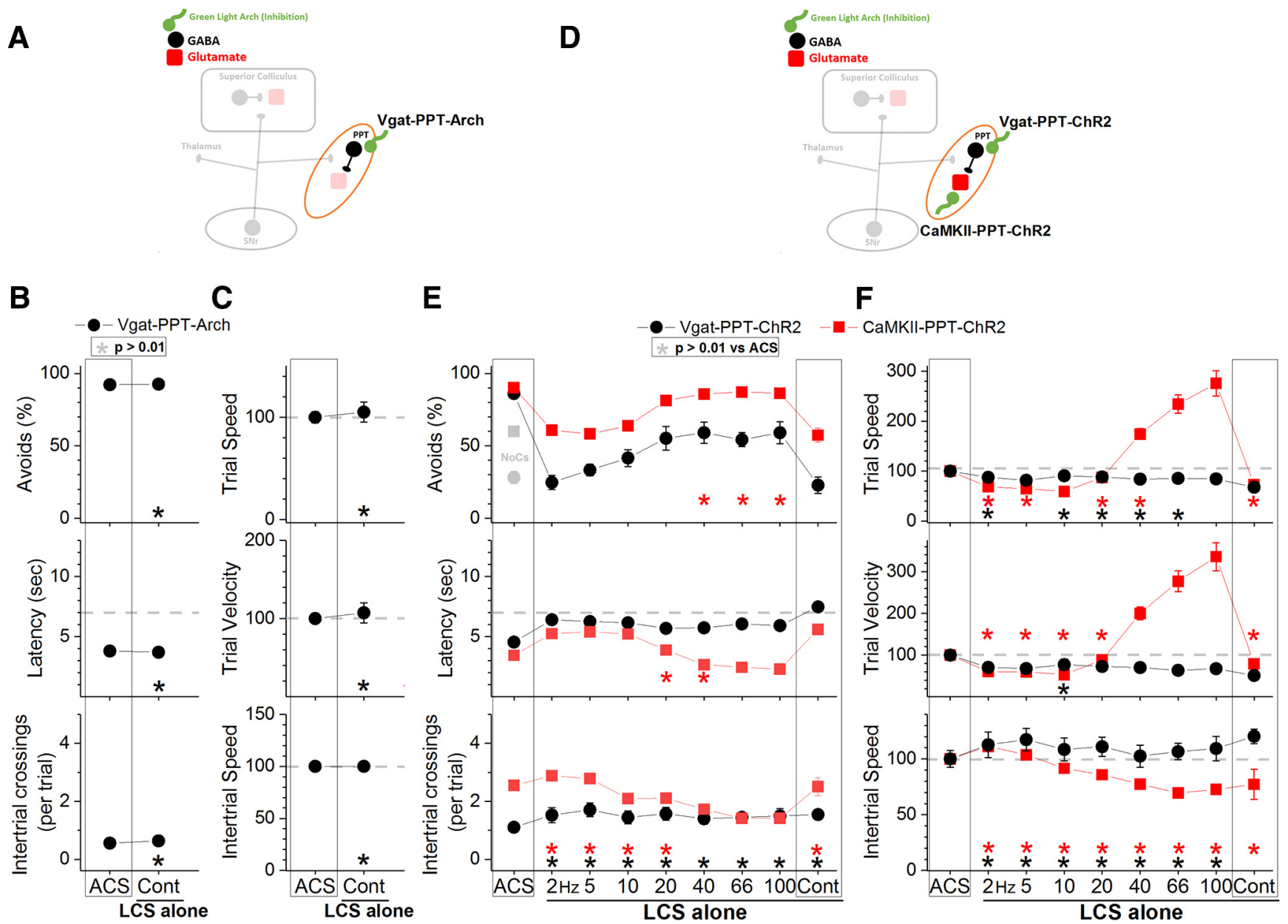

Figure 10. Effect of activating or inhibiting cells in the PPT on driving avoidance responses in the absence of the ACS. $A$, Schematic of the PPT cells inhibited in the experiments shown in $B$ and $C$. $B$, Effect of green light applied in the PPT on LCS alone trials for animals that express Arch in GABAergic (Vgat-PPT-Arch) cells in the PPT. The asterisks highlight nonsignificant difference versus ACS trials. C, Trial speed, trial velocity, and intertrial speed for the data in $\boldsymbol{B}$. D. Schematic of the PPT cells activated in the experiments shown in $\boldsymbol{E}$ and $\boldsymbol{F}$. $\boldsymbol{E}$, Effect of blue light applied in the PPT on LCS alone trials for animals that express ChR2 in GABAergic (Vgat-PPT-ChR2) or putative glutamatergic (CaMKII-PPT-ChR2) cells in the PPT. The asterisks highlight nonsignificant difference versus ACS trials. $\boldsymbol{F}$, Trial speed, trial velocity, and intertrial speed for the data in $\boldsymbol{E}$.

blue light trains at $20 \mathrm{~Hz}$, the response speed $(p=0.8)$, velocity $(p=0.8)$ and latency $(p=0.06)$ were equivalent to avoidance responses driven by the ACS. Increasing the blue light frequency $>20 \mathrm{~Hz}$ lead to large increases in speed as per the ACS+LCS trials. As a consequence of these fast avoidance responses, the performance in the task changed during ACS alone trials, so that the overall number of intertrial crossings typically increased. However, the number of intertrial crossings and the intertrial speed decreased immediately after trials in which fast avoidance responses occurred. In other words, the animals paused during the intertrial interval after each fast avoidance response caused by blue light trains $>20 \mathrm{~Hz}$. Moreover, elicitation of these fast shuttling responses did not require learning; naive animals innately expressed them (shuttled to the other compartment when presented with this optogenetic stimulation) during excitation of putative glutamatergic cells in PPT (effects are similar to Fig. $10 E, F$, filled red squares).

The PPT contains a population of cholinergic cells intermixed with putative glutamatergic and GABAergic cells $(\mathrm{H}$. L. Wang and Morales, 2009). To determine the effects of exciting these cholinergic cells on ACS + LCS and LCS alone trials we used ChatPPT-ChR2 mice [cross of Chat-cre (Jax 028861) and Ai32 mice to express ChR2 in cholinergic cells] with optical fibers implanted in PPT. Excitation of cholinergic cells in the PPT of Chat-PPT-
ChR2 mice with the regular power level of blue light (2-3 mW) had no effect on avoidance responses, so we tested the higherpower level ( $6 \mathrm{~mW}$, range: 6$)$. The higher level of blue light (Fig. 12, black closed circles; 15 sessions in 3 mice) also had no effect on the percentage of avoidance responses in ACS + LCS trials using any of the light frequency trains (Tukey, $p>0.82$ for each light train vs ACS) or continuous light $(p=0.99)$. The same highpower optogenetic stimulation was also ineffective at driving avoidance responses in LCS alone trials (Fig. 12, green open circles; 15 sessions in 3 mice). Although it is worth noting that continuous blue light at this high-power level drove avoidance responses at a rate $(60 \pm 7 \%)$ that was above No CS trials (26.6 \pm $5 \%)$, but this rate was still significantly below ACS trials (86.07 \pm 1.5 ; Tukey, $p=0.01$, ACS vs Cont). The other LCS alone blue light stimuli (2-100 Hz trains) did not differ from No CS trials and were significantly below ACS trials (Tukey, $p<0.01$ for each light train vs ACS). Thus, it is primarily the excitation of putative glutamatergic cells in PPT, not of cholinergic or GABAergic cells, which drives avoidance responses.

In conclusion, putative glutamatergic cells in PPT are required for signaled active avoidance and their excitation innately causes shuttling that resembles avoidance responses or more rapid escape responses depending on the excitation frequency. 
A
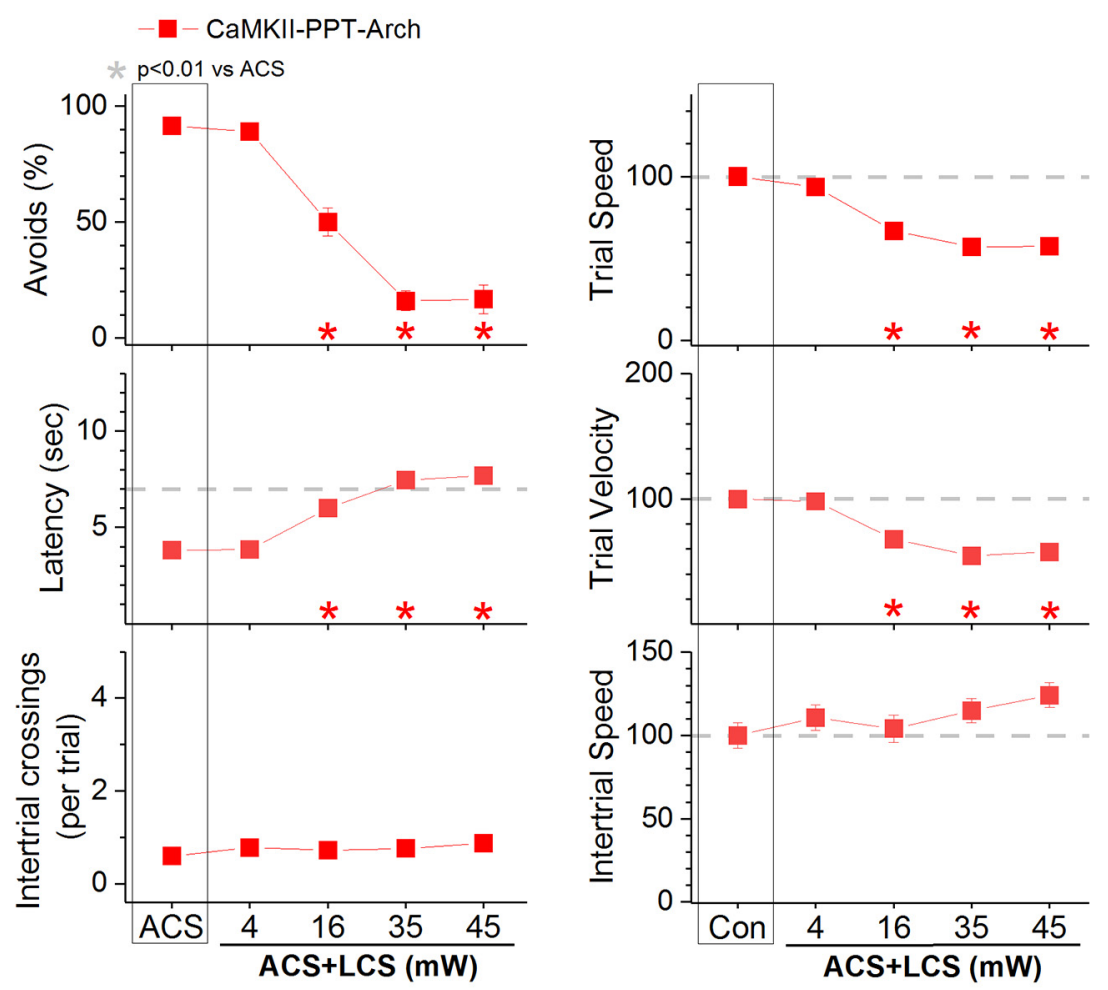

B
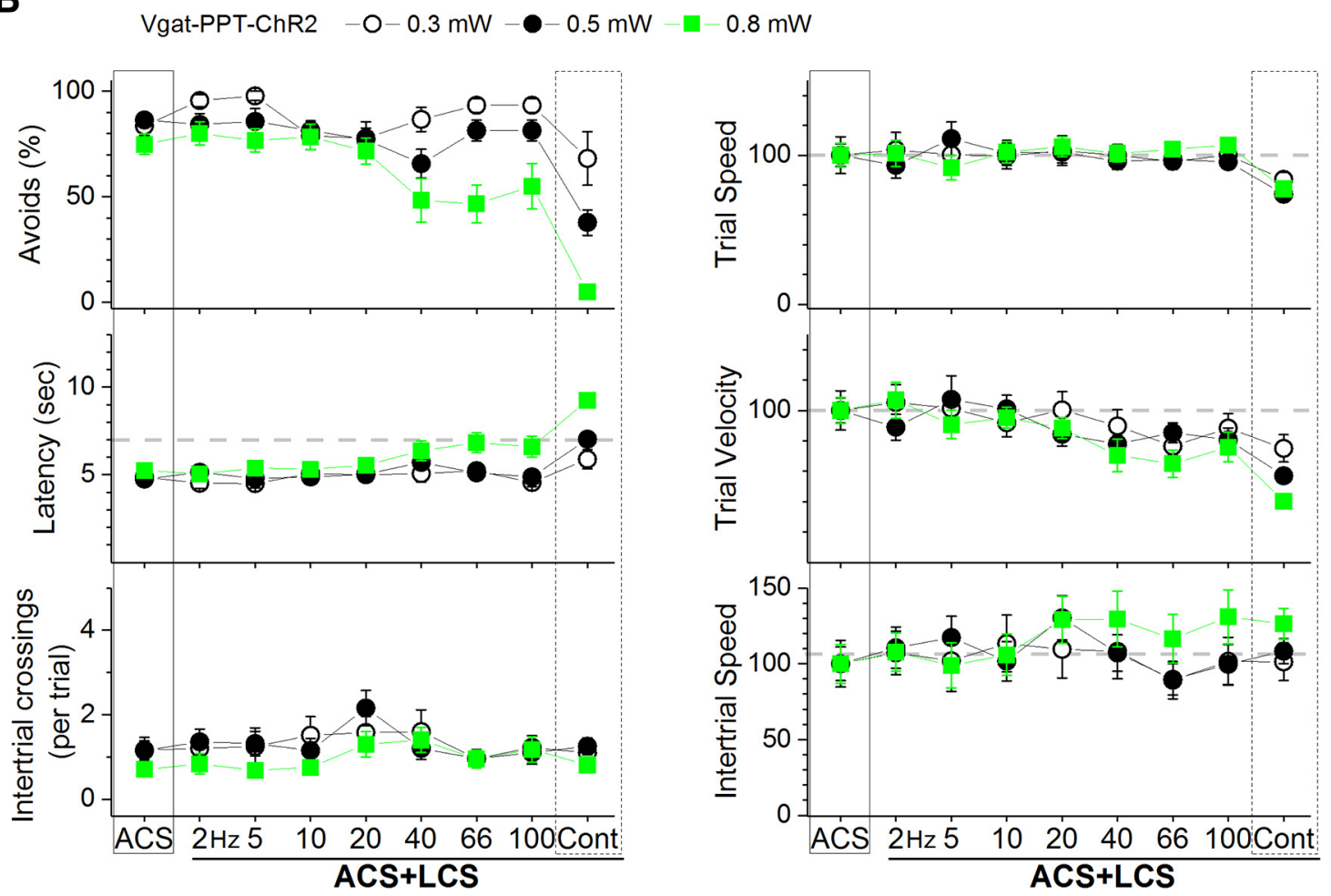

Figure 11. Effect of different light powers on avoidance responses driven by the ACS in Vgat-PPT-Arch and CaMKII-PPT-ChR2 mice. A, Effect of green light applied in the PPT at different powers on ACS + LCS trials for animals that express Arch in putative glutamatergic PPT cells (CaMKII-PPT-Arch). B, Effect of blue light applied in the PPT at different powers on ACS + LCS trials for animals that express ChR2 in GABAergic PPT cells (Vgat-PPT-ChR2).

\section{Discussion}

Inhibition of putative glutamatergic PPT cells or excitation of local GABAergic PPT cells, at levels that preserve escape responses, abolish signaled active avoidance responses. Activation of inhibitory synaptic inputs from $\mathrm{SNr}$ also suppress the genera- tion of avoidance responses in PPT. Conversely, excitation of putative glutamatergic PPT cells, or inhibition of GABAergic PPT cells, can be tuned to drive avoidance responses. For instance, excitation of putative glutamatergic PPT cells with blue light at $\sim 20 \mathrm{~Hz}$ (1 ms pulses) drives shuttling responses at a latency, speed, and 
velocity that resemble avoidance responses driven by an ACS. The PPT is a crucial junction for signaled active avoidance that can integrate excitatory and inhibitory inputs from various sources to control the generation of signaled active avoidance responses.

\section{The superior colliculus and CS processing}

The superior colliculus is a well known sensorimotor structure that has an established role in detecting sensory stimuli that require immediate action, such as in orienting behaviors (Wurtz and Hikosaka, 1986; Dean et al., 1989; Stein and Meredith, 1993; Hikosaka et al., 2000; Cohen et al., 2008; Felsen and Mainen, 2008; Gandhi and Katnani, 2011; Krauzlis et al., 2013). Moreover, superior colliculus cells fire during signaled active avoidance responses (Cohen and Castro-Alamancos, 2010b), and their excitation drives escape responses (Shang et al., 2015; Wei et al., 2015). However, inhibition of putative glutamatergic cells in the superior colliculus does not block signaled active avoidance, which agrees with the effects of superior colliculus lesions (Cohen and Castro-Alamancos, 2007). Consistent with a role in CS processing and detection, we found that a wide variety of modulations of putative glutamatergic or GABAergic cells in the superior colliculus were effective conditioned signals to drive avoidance responses. In particular, excitation of putative glutamatergic cells was the best stimulus within the superior colliculus to drive shuttling responses that resemble avoidance responses; although at the highest stimulation frequencies, these responses were less intense in speed than those that were driven from PPT. Thus, the superior colliculus, while not required for signaled active avoidance, seems to function as one of the redundant (parallel) sensory relays that can provide the PPT with CS-related signals necessary to drive avoidance responses. Indeed, many brainstem and forebrain areas that process sensory stimulation related to the CS project to $\mathrm{PPT}$, such as the superior colliculus, inferior colliculus, and sensory cortices, including the auditory cortex (Martinez-Gonzalez et al., 2011; Roseberry et al., 2016). These pathways can provide redundant CS-related signals to PPT, which could explain why signaled active avoidance responses remain intact after one of these sensory pathways is lesioned, and why synergy between them is required when CS salience is weak (Cohen and Castro-Alamancos, 2007, 2010a). Knowing that PPT is an essential component of the circuitry that expresses avoidance responses will allow us to determine whether the synergistic integration of weak inputs from various sensory regions required to detect weak CS stimuli occurs in PPT.

\section{PPT has a critical role in signaled active avoidance}

The midbrain locomotor region (MLR), which includes PPT, cuneiform and mesencephalic reticular nuclei, regulates spontaneous locomotion (Shik et al., 1966; Skinner and Garcia-Rill,
1984; Ryczko and Dubuc, 2013; Roseberry et al., 2016; Xiao et al., 2016; Caggiano et al., 2018). We highlight the PPT because the optical fibers that blocked signaled active avoidance responses with minute power levels were squarely located in between the middle and dorsal portions of PPT. Moreover, SNr fibers appear to preferentially target PPT within the MLR. However, we do not discount the possibility that putative glutamatergic cells in the other MLR nuclei (e.g., cuneiform) can have similar roles as PPT.

Our results show that levels of PPT inhibition that completely block signaled active avoidance do not interfere with the ability of the animals to escape the US. Thus, we specifically blocked the ability of the CS, but not of the US, to generate a locomotor action. When PPT cells were directly modulated, there were typically effects on trial speed, and especially trial velocity, consistent with a role of PPT in setting spontaneous locomotion speed (Roseberry et al., 2016; Caggiano et al., 2018), but during these manipulations animals still responded effectively by rapidly escaping the US. It is not clear whether escape responses employ the same expression pathways as avoidance responses in PPT; this was not an aim of the study. However, escape responses to the US were typically affected when we excited GABAergic cells or GABAergic afferents in PPT, not when we directly inhibited putative glutamatergic cells in PPT. 
Activation of GABAergic cells may lead to inhibition of other targets of these cells outside of PPT or may cause a stronger inhibition of PPT cells than direct inhibition with eArchT3.0. Nevertheless, if PPT mediates both avoidance and escape responses, our results clearly show that blocking escape responses requires much stronger inhibition of PPT cells than blocking avoidance responses, which indicates that the synaptic inputs in PPT that drive avoidance responses are much weaker than those that drive escape responses.

The PPT is composed of cholinergic, GABAergic, and glutamatergic neuronal subtypes. Cholinergic cells are concentrated in the dorsal and posterior PPT, and GABAergic cells are concentrated in the ventral and anterior PPT (Mena-Segovia et al., 2009; X. Wang and Morales, 2009). Interestingly, chemical lesions of PPT in rats have been shown to impair learning of signaled active avoidance (the effects of PPT lesions on expression was not tested); an effect that was attributed to cholinergic deficits (Fujimoto et al., 1989). We found that the excitation of putative glutamatergic PPT cells, and not of GABAergic or cholinergic PPT cells, drives active avoidance responses. Cholinergic cells may have a modulatory or supporting role because their excitation with strong optogenetic stimuli could drive some avoidance responses. Instead, both GABAergic and putative glutamatergic PPT cells have a critical role in signaled active avoidance responses, which may be fulfilled by both ascending and descending projections from PPT (Capelli et al., 2017; Mena-Segovia and Bolam, 2017; Arber and Costa, 2018). In particular, the descending projections ideally target locomotion-generating regions in the caudal brainstem. The ascending projections target back the basal ganglia forming feedback loops that may be useful to regulate the control exerted by $\mathrm{SNr}$ over avoidance responses in PPT.

\section{A circuit model for signaled active avoidance}

The results highlight a model in which afferents from CS processing areas provide excitatory inputs to PPT that drive avoidance responses (e.g., superior colliculus), whereas inhibitory inputs from other regions (e.g., $\mathrm{SNr}$ ) have a gating (permissive) role that allow avoidance responses to be expressed. The balance between the driving and gating inputs in putative glutamatergic PPT cells can finely regulate the expression of signaled avoidance responses. Learning may occur through modifications in the strength of the driving inputs, gating inputs or both, as a consequence of local changes within PPT or remote changes in the afferent areas. This framework is useful to explore, in future experiments, the specific role of different PPT afferent areas involved in signaled active avoidance. For instance, PPT receives direct inputs from the amygdala and ventral striatum (Roseberry et al., 2016), which have been proposed to have significant roles in signaled active avoidance (Roozendaal et al., 1993; Choi et al., 2010; Lichtenberg et al., 2014; Bravo-Rivera et al., 2015; Ramirez et al., 2015). Moreover, understanding how neural circuits normally mediate this learned, CS-signaled, locomotor action is important because alterations in these circuits may lead to symptoms present in psychiatric (pathological avoidance in anxiety disorders) and neurological disorders (cue-driven movement responsiveness in Parkinson's disease).

\section{References}

Arber S, Costa RM (2018) Connecting neuronal circuits for movement. Science 360:1403-1404.

Bentivoglio M, van der Kooy D, Kuypers HG (1979) The organization of the efferent projections of the substantia nigra in the rat: a retrograde fluorescent double labeling study. Brain Res 174:1-17.

Betley JN, Xu S, Cao ZF, Gong R, Magnus CJ, Yu Y, Sternson SM (2015)
Neurons for hunger and thirst transmit a negative-valence teaching signal. Nature 521:180-185.

Blanchard DC, Griebel G, Blanchard RJ (2001) Mouse defensive behaviors: pharmacological and behavioral assays for anxiety and panic. Neurosci Biobehav Rev 25:205-218.

Bolles RC (1970) Species-specific defense reactions and avoidance learning. Psychol Rev 77:32-48.

Bolles RC, Stokes LW, Younger MS (1966) Does CS termination reinforce avoidance behavior? J Comp Physiol Psychol 62:201-207.

Bravo-Rivera C, Roman-Ortiz C, Montesinos-Cartagena M, Quirk GJ (2015) Persistent active avoidance correlates with activity in prelimbic cortex and ventral striatum. Front Behav Neurosci 9:184.

Caggiano V, Leiras R, Goñi-Erro H, Masini D, Bellardita C, Bouvier J, Caldeira V, Fisone G, Kiehn O (2018) Midbrain circuits that set locomotor speed and gait selection. Nature 553:455-460.

Capelli P, Pivetta C, Soledad Esposito M, Arber S (2017) Locomotor speed control circuits in the caudal brainstem. Nature 551:373-377.

Cebrián C, Parent A, Prensa L (2005) Patterns of axonal branching of neurons of the substantia nigra pars reticulata and pars lateralis in the rat. J Comp Neurol 492:349-369.

Chevalier G, Deniau JM (1990) Disinhibition as a basic process in the expression of striatal functions. Trends Neurosci 13:277-280.

Choi JS, Cain CK, LeDoux JE (2010) The role of amygdala nuclei in the expression of auditory signaled two-way active avoidance in rats. Learn Mem 17:139-147.

Chow BY, Han X, Dobry AS, Qian X, Chuong AS, Li M, Henninger MA, Belfort GM, Lin Y, Monahan PE, Boyden ES (2010) High-performance genetically targetable optical neural silencing by light-driven proton pumps. Nature 463:98-102.

Cohen JD, Castro-Alamancos MA (2007) Early sensory pathways for detection of fearful conditioned stimuli: tectal and thalamic relays. J Neurosci 27:7762-7776.

Cohen JD, Castro-Alamancos MA (2010a) Detection of low salience whisker stimuli requires synergy of tectal and thalamic sensory relays. J Neurosci 30:2245-2256.

Cohen JD, Castro-Alamancos MA (2010b) Neural correlates of active avoidance behavior in superior colliculus. J Neurosci 30:8502-8511.

Cohen JD, Hirata A, Castro-Alamancos MA (2008) Vibrissa sensation in superior colliculus: wide-field sensitivity and state-dependent cortical feedback. J Neurosci 28:11205-11220.

Dean P, Redgrave P, Westby GW (1989) Event or emergency? Two response systems in the mammalian superior colliculus. Trends Neurosci 12:137147.

Di Chiara G, Porceddu ML, Morelli M, Mulas ML, Gessa GL (1979) Evidence for a GABAergic projection from the substantia nigra to the ventromedial thalamus and to the superior colliculus of the rat. Brain Res 176:273-284.

Felsen G, Mainen ZF (2008) Neural substrates of sensory-guided locomotor decisions in the rat superior colliculus. Neuron 60:137-148.

Fujimoto K, Yoshida M, Ikeguchi K, Niijima K (1989) Impairment of active avoidance produced after destruction of pedunculopontine nucleus areas in the rat. Neurosci Res 6:321-328.

Gandhi NJ, Katnani HA (2011) Motor functions of the superior colliculus. Annu Rev Neurosci 34:205-231.

Gulcebi MI, Ketenci S, Linke R, Hacıoğlu H, Yanalı H, Veliskova J, Moshé SL, Onat F, Çavdar S (2012) Topographical connections of the substantia nigra pars reticulata to higher-order thalamic nuclei in the rat. Brain Res Bull 87:312-318.

Hikosaka O, Takikawa Y, Kawagoe R (2000) Role of the basal ganglia in the control of purposive saccadic eye movements. Physiol Rev 80:953-978.

Hormigo S, Vega-Flores G, Castro-Alamancos MA (2016) Basal ganglia output controls active avoidance behavior. J Neurosci 36:10274-10284.

Kamin LJ, Brimer CJ, Black AH (1963) Conditioned suppression as a monitor of fear of the CS in the course of avoidance training. J Comp Physiol Psychol 56:497-501.

Krauzlis RJ, Lovejoy LP, Zénon A (2013) Superior colliculus and visual spatial attention. Annu Rev Neurosci 36:165-182.

Lalive AL, Lien AD, Roseberry TK, Donahue CH, Kreitzer AC (2018) Motor thalamus supports striatum-driven reinforcement. eLife 7:e34032.

Lammel S, Lim BK, Ran C, Huang KW, Betley MJ, Tye KM, Deisseroth K, Malenka RC (2012) Input-specific control of reward and aversion in the ventral tegmental area. Nature 491:212-217. 
LeDoux JE, Moscarello J, Sears R, Campese V (2017) The birth, death and resurrection of avoidance: a reconceptualization of a troubled paradigm. Mol Psychiatry 22:24-36.

Lichtenberg NT, Kashtelyan V, Burton AC, Bissonette GB, Roesch MR (2014) Nucleus accumbens core lesions enhance two-way active avoidance. Neuroscience 258:340-346.

Martinez-Gonzalez C, Bolam JP, Mena-Segovia J (2011) Topographical organization of the pedunculopontine nucleus. Front Neuroanat 5:22.

Mattis J, Tye KM, Ferenczi EA, Ramakrishnan C, O'Shea DJ, Prakash R, Gunaydin LA, Hyun M, Fenno LE, Gradinaru V, Yizhar O, Deisseroth K (2011) Principles for applying optogenetic tools derived from direct comparative analysis of microbial opsins. Nat Methods 9:159-172.

Mena-Segovia J, Bolam JP (2017) Rethinking the pedunculopontine nucleus: from cellular organization to function. Neuron 94:7-18.

Mena-Segovia J, Bolam JP, Magill PJ (2004) Pedunculopontine nucleus and basal ganglia: distant relatives or part of the same family? Trends Neurosci 27:585-588.

Mena-Segovia J, Micklem BR, Nair-Roberts RG, Ungless MA, Bolam JP (2009) GABAergic neuron distribution in the pedunculopontine nucleus defines functional subterritories. J Comp Neurol 515:397-408.

Mineka S (1979) The role of fear in theories of avoidance learning, flooding, and extinction. Psychol Bull 86:985-1010.

Mineka S, Hendersen RW (1985) Controllability and predictability in acquired motivation. Annu Rev Psychol 36:495-529.

Mowrer OH (1960) Learning theory and behavior. New York: Wiley.

Ramirez F, Moscarello JM, LeDoux JE, Sears RM (2015) Active avoidance requires a serial basal amygdala to nucleus accumbens shell circuit. J Neurosci 35:3470-3477.

Rescorla RA, Solomon RL (1967) Two-process learning theory: relationships between Pavlovian conditioning and instrumental learning. Psychol Rev 74:151-182.

Richards CD, Shiroyama T, Kitai ST (1997) Electrophysiological and immunocytochemical characterization of GABA and dopamine neurons in the substantia nigra of the rat. Neuroscience 80:545-557.

Roozendaal B, Koolhaas JM, Bohus B (1993) The central amygdala is involved in conditioning but not in retention of active and passive shock avoidance in male rats. Behav Neural Biol 59:143-149.

Roseberry TK, Lee AM, Lalive AL, Wilbrecht L, Bonci A, Kreitzer AC (2016) Cell-type-specific control of brainstem locomotor circuits by basal ganglia. Cell 164:526-537.

Ryczko D, Dubuc R (2013) The multifunctional mesencephalic locomotor region. Curr Pharm Des 19:4448-4470.

Seligman MEP, Johnston JA, McGuigan FJ, Lumsden DB (1973) A cognitive theory of avoidance learning. In: Contemporary approaches to conditioning and learning. New York: Wiley.

Shabel SJ, Proulx CD, Trias A, Murphy RT, Malinow R (2012) Input to the lateral habenula from the basal ganglia is excitatory, aversive, and suppressed by serotonin. Neuron 74:475-481.

Shang C, Liu Z, Chen Z, Shi Y, Wang Q, Liu S, Li D, Cao P (2015) A parvalbumin-positive excitatory visual pathway to trigger fear responses in mice. Science 348:1472-1477.

Shik ML, Severin FV, Orlovskiǔ GN (1966) Control of walking and running by means of electric stimulation of the midbrain [in Russian]. Biofizika 11:659-666.

Skinner RD, Garcia-Rill E (1984) The mesencephalic locomotor region (MLR) in the rat. Brain Res 323:385-389.

Stein BE, Meredith MA (1993) The merging of the senses. Cambridge, MA: MIT.

Tan KR, Yvon C, Turiault M, Mirzabekov JJ, Doehner J, Labouèbe G, Deisseroth K, Tye KM, Lüscher C (2012) GABA neurons of the VTA drive conditioned place aversion. Neuron 73:1173-1183.

Tepper JM, Martin LP, Anderson DR (1995) GABAA receptor-mediated inhibition of rat substantia nigra dopaminergic neurons by pars reticulata projection neurons. J Neurosci 15:3092-3103.

Tovote P, Fadok JP, Lüthi A (2015) Neuronal circuits for fear and anxiety. Nat Rev Neurosci 16:317-331.

Wang HL, Morales M (2009) Pedunculopontine and laterodorsal tegmental nuclei contain distinct populations of cholinergic, glutamatergic and GABAergic neurons in the rat. Eur J Neurosci 29:340-358.

Wang X, Zhang C, Szábo G, Sun QQ (2013) Distribution of CaMKII $\alpha$ expression in the brain in vivo, studied by CaMKIIalpha-GFP mice. Brain Res 1518:9-25.

Wei P, Liu N, Zhang Z, Liu X, Tang Y, He X, Wu B, Zhou Z, Liu Y, Li J, Zhang Y, Zhou X, Xu L, Chen L, Bi G, Hu X, Xu F, Wang L (2015) Processing of visually evoked innate fear by a non-canonical thalamic pathway. Nat Commun 6:6756.

Wiegert JS, Mahn M, Prigge M, Printz Y, Yizhar O (2017) Silencing neurons: tools, applications, and experimental constraints. Neuron 95:504-529.

Wurtz RH, Hikosaka O (1986) Role of the basal ganglia in the initiation of saccadic eye movements. Prog Brain Res 64:175-190.

Xiao C, Cho JR, Zhou C, Treweek JB, Chan K, McKinney SL, Yang B, Gradinaru V (2016) Cholinergic mesopontine signals govern locomotion and reward through dissociable midbrain pathways. Neuron 90:333-347.

Yilmaz M, Meister M (2013) Rapid innate defensive responses of mice to looming visual stimuli. Curr Biol 23:2011-2015. 